\title{
A MINIMIZAÇÃO DO CUSTO DO PRODUTO AGRÍCOLA EM ENSAIOS DE ADUBAÇÃO MINERAL
}

\author{
CRISTIÁN ANDRÉS CARRANZA \\ Engenheiro Florestal
}

Orientador: Prof. Dr. F. PIMENTEL-GOMES

Dissertação apresentada à Escola Superior de Agricultura "Luiz de Queiroz", Universidade de São Paulo, para obtenção do título de Mestre em Agronomia, Área de Concentração: Estatística e Experimentação Agronomica.

PIRACICABA

Estado de São Paulo - Brasil

Setembro - 1998 
Dados Internacionais de Catalogação na Publicação (CIP) DIVISÃO DE BIBLIOTECA E DOCUMENTAÇÃO - Campus "Luiz de Queiroz"/USP

\author{
Carranza, Cristián Andrés \\ A minimizaçăo do custo do produto agricola em ensaios de adubação mineral / \\ Cristián Andrés Carranza. - - Piracicaba, 1998. \\ 47 p. : il. \\ Dissertação (mestrado) - Escola Superior de Agricultura Luiz de Queiroz, 1998. \\ Bibliografia. \\ 1. Adubação 2. Análise da variância 3. Custo de produção 4. Produtividade \\ agricola 5. Produto agricola I. Título
}

CDD 338.2764 


\section{A MINHA MÃE e ao seu amor incondicional.}

Às minhas irmãs, Claudia, Gaby e Graciela, que, como eu, voaram do ninho há tempo, para encontrar um lugar no mundo.

A Vangelis, Rick Deckard e Luke Skywalker:

... 'I've seen things you people wouldn't believe. Attack ships on fire off the shoulders of Orion. I've watched C-beams, glittering in the darkness of Tannhausser Gate.

All of those moments will be lost in time, like tears in the rain." R.B. 


\section{AGRADECIMENTOS}

\section{Ao professor doutor F. Pimentel-Gomes, o $\delta \epsilon \iota \iota^{\prime} \xi \alpha \varsigma$.}

Ao professor Rafael Boggio Ronceros, do Curso de Cálculo Estadístico y Biometría, de la Facultad de Ciencias Agrarias y Forestales de la Universidad Nacional de La Plata, o meu mentor.

A Maria Helena, que apareceu quando tudo era não-significativo.

A Iza, que me ensinou a ver onde poucos vêem.

Aos meus colegas de mestrado: Afrânio, Adilson, Wilson, Sandra, Jeanete e Helena, pois eles levaram à normalidade um curso cuja distribuição era Cauchy.

À amiga Sílvia e ao seu mascote CNX.

Aos professores do Departamento de Matemática e Estatística, por me acordarem.

Ao Jorge, pela amizade.

À FAPESP, que conseguiu fazer com que meu único problema seja desenvolver e escrever este trabalho. 


\section{SUMÁRIO}

\section{Página}

LISTA DE FIGURAS $\ldots \ldots \ldots \ldots \ldots \ldots$ iii

LISTA DE TABELAS $\ldots \ldots \ldots \ldots \ldots \ldots \ldots \ldots$ iv

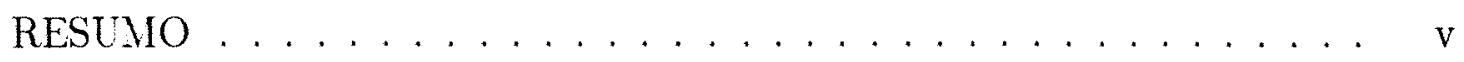

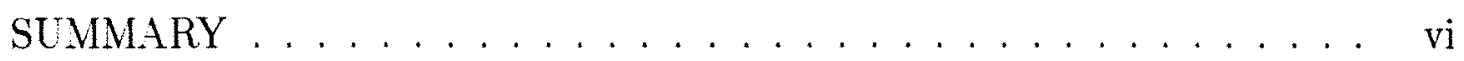

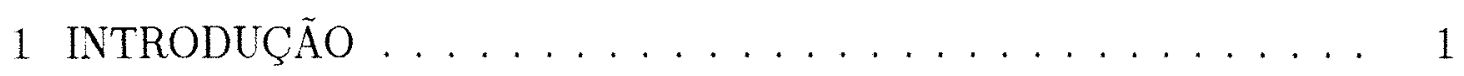

2 REVISÃo DE LiTERATURA . . . . . . . . . . . . . . 6

3 MATERIAL E MÉtodod . . . . . . . . . . . . . . . . . . . . . 12

3.1 Material . . . . . . . . . . . . . . . . . . . . . 12

3.2 Vétodos . . . . . . . . . . . . . . . . . . . . . . . . . . 14

4 RESULTADOS E DISCUSSÃO _ . . . . . . . . . . . . 17

4.1 Análise da Variância . . . . . . . . . . . . . . . . . . . 17

4.2 Estudo com Duas Variáveis . . . . . . . . . . . . . . 22

4.2 .1 Análise da Variância . . . . . . . . . . . . . . . . . . . 22

4.2.2 Estimação e Estudo dos Parâmetros da Superfície $\mathrm{Y}(\mathrm{N}, \mathrm{K}) \ldots \ldots$ 
4.2.3 A Função $\mathrm{G}(\mathrm{N}, \mathrm{K})$ de Produtividade por Real . . . . . . . . . . . . 26

4.2 .4 Análise Canônica de $\mathrm{G}(\mathrm{N}, \mathrm{K}) \ldots \ldots \ldots$

4.3 Estudo com Três Variáveis . . . . . . . . . . . . . . . . . 31

4.3.1 Análise da Variância . . . . . . . . . . . . . . . . . . . 31

4.3.2 Estimação e Estudo dos Parâmetros da Superfície Y(N, P, K) . . . . 33

4.3.3 A Função G(N, P, K) de Produtividade por Real . . . . . . . . . . . . 34

4.3.4 Análise Canônica de $\mathrm{G}(\mathrm{N}, \mathrm{P}, \mathrm{K}) \ldots \ldots \ldots$

4.4 Validação . . . . . . . . . . . . . . . . . . . . . . 39

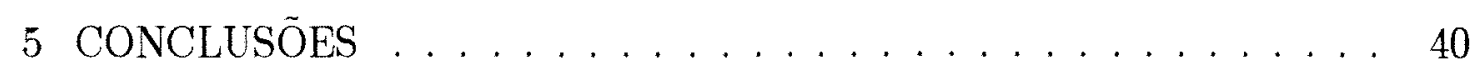

REFERÊNCIAS BIBLIOGRÁFICAS . . . . . . . . . . . . . . . . 42

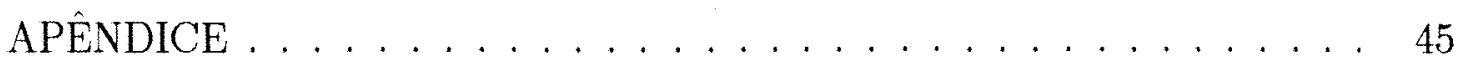




\section{LISTA DE FIGURAS}

Página

1 Modelo geral: dispersão dos resíduos padronizados . . . . . . . . . . . 18

$2 \mathrm{Y}(\mathrm{N}, \mathrm{K})$ : Dispersão dos resíduos padronizados . . . . . . . . . 25

3 Superfície de resposta para a produtividade de cana-de-açúcar. . . . 26

4 Superfície $\mathrm{H}_{1}(\mathrm{~N}, \mathrm{~K})$ que aproxima $\mathrm{G}(\mathrm{N}, \mathrm{K})$ na região experimental. . 28

5 Pontos estimados da função $\mathrm{G}(\mathrm{N}, \mathrm{K}) \ldots \ldots \ldots$

$6 \mathrm{Y}(\mathrm{N}, \mathrm{P}, \mathrm{K})$ : Dispersão dos resíduos padronizados . . . . . . . . . 34

7 Gráficos de contorno do polinômio $\mathrm{H}_{1}(\mathrm{~N}, \mathrm{P}, \mathrm{K}) \ldots \ldots \ldots$

8 Esquema da estratégia seguida no processo iterativo . . . . . . . . 38 


\section{LISTA DE TABELAS}

Página

1 Quadrados Médios Residuais . . . . . . . . . . . . . . . . . 13

2 Preços dos adubos $\ldots \ldots \ldots \ldots \ldots \ldots$

3 Modelo geral: análise da variância . . . . . . . . . . . . . . . 17

4 Desdobramento do modelo geral: análise da variância . . . . . . . . . 21

$5 \quad \mathrm{Y}(\mathrm{N}, \mathrm{K})$ : análise da variância $\ldots \ldots \ldots \ldots$

$6 \quad \mathrm{Y}(\mathrm{N}, \mathrm{K})$ : teste de falta de ajuste $\ldots \ldots \ldots \ldots \ldots$

$7 \quad$ Valores de $\mathrm{G}$ para os níveis de $\mathrm{N}$ e K . . . . . . . . . . 27

$8 \quad \mathrm{Y}(\mathrm{N}, \mathrm{P}, \mathrm{K})$ : análise da variância $\ldots \ldots \ldots \ldots$

$9 \quad \mathrm{Y}(\mathrm{N}, \mathrm{P}, \mathrm{K}):$ teste de falta de ajuste $\ldots \ldots \ldots \ldots \ldots$

10 Valores de $\mathrm{G}$ para os níveis de $\mathrm{N}, \mathrm{P}$ e $\mathrm{K} \ldots \ldots$

11 Rendimentos por experimento $(\mathrm{kg} / \mathrm{ha}) \ldots \ldots \ldots \ldots$ 


\title{
A MINIMIZAÇÃO DO CUSTO DO PRODUTO AGRÍCOLA EM ENSAIOS DE ADUBAÇÃO MINERAL
}

\author{
Autor: CRISTIÁN ANDRÉS CARRANZA \\ Orientador: Prof. Dr. F. PIMENTEL-GOMES
}

\section{RESUMO}

O objetivo deste trabalho foi o de determinar as doses de nutrientes que minimizam o custo do produto agrícola em ensaios de adubação. Para isso, foi desenvolvida uma função $\mathrm{G}$, a duas e, posteriormente, a três variáveis, obtida pelo quociente de um polinômio de segundo grau ajustado à produtividade obtida a partir de dados experimentais, e uma função linear dos custos envolvidos. Um outro polinômio de segundo grau foi ajustado iterativamente a fim de aproximar o comportamento dessa função G. Fez-se a anâlise canônica desta superfície a fim de determinar o ponto de máximo, que exprime a máxima quantidade de produto obtida por unidade de moeda. Analogamente, o ponto de mínimo de $\mathrm{G}^{-1}$ exprime o custo mínimo por unidade de produto agrícola. Tanto no caso de duas como de três variáveis, a função $\mathrm{G}$ foi muito bem aproximada por um polinômio de segundo grau e a metodologia desenvolvida demonstrou resolver bem o problema. A convergência do processo iterativo demostrou ser rápida também. Algumas considerações são realizadas em relação ao uso do pacote estatístico computacional SAS para resolver este tipo de problema. 


\title{
THE MINIMIZATION OF AN AGRICULTURAL PRODUCT COST FUNCTION IN FERTILIZER EXPERIMENTS
}

\author{
Author: CRISTIÁN ANDRÉS CARRANZA \\ Adviser: Prof. Dr. F. PIMENTEL-GOMES
}

\section{SUMMARY}

The aim of this work was the determination of the nutrient doses that minimize the product cost in fertilizer experiments. In doing so, a function $\mathrm{G}$ was developed, firstly with two explanatory variables and with three afterwards. This function is defined as the ratio between a second order polynomial, fitted to crop productivity taken from experimental data, and a linear function of associated costs. A new second order polynomial was fitted iteratively, in order to approach the behavior of the $\mathrm{G}$ function, and the corresponding canonical analysis was performed to determine its maximum point. That optimum yields the maximum amount of product obtained by monetary unit and, analogously, the minimum point of $\mathrm{G}^{-1}$ yields the minimum cost by unit produced. Either in the two or in the three variate case, the $\mathrm{G}$ function was very well approached by a second order polynomial, and the methodology presented dealt well with the problem and converged rapidly. Some remarks are presented with regard to the use of the SAS software as a tool for dealing with such a problem. 


\section{INTRODUÇÃO}

A produtividade de um cultivo agrícola pode ser aproximada mediante o uso de um modelo matemático. Como o processo subjacente não é completamente conhecido, o experimentador deve aproximar a função desconhecida através de um modelo empírico. Usualmente, a função escolhida é um polinômio de segundo grau a uma ou mais variáveis independentes. No caso de duas ou mais variáveis independentes, a representação geométrica deste modelo é chamada de Superfície de Resposta (Myers \& Montgomery, 1995).

No caso dos experimentos de adubação, a função pode ser representada como

$$
\eta=\varphi\left(X_{1}, X_{2}, \ldots, X_{n}\right),
$$

sendo $X_{1}, X_{2}, \ldots, X_{n}$ os níveis dos nutrientes utilizados, e $\eta$ a produtividade. Ela é estimada a partir de dados numéricos que exprimam o comportamento da produção relativamente a essas doses de nutrientes. Em termos mais agronômicos, essa função é chamada Função de Produção. Assim, para a u-ésima combinação dos níveis dos fatores $(u=1, \ldots N)$, fica:

$$
\eta_{u}=\varphi\left(X_{1 u}, \ldots X_{k u}\right) .
$$


Devido ao efeito de fatores não controlados, a resposta observada $y_{u}$ varia entre observações, com média $\mu_{u}$ e variância $\sigma^{2}$, com distribuição que se pode, em geral, considerar normal.

Segundo Heady \& Dillon (1961), essas funções, representadas por equaçōes de regressão, relacionam a produtividade física de um cultivo agrícola com um ou mais fatores de interesse. Geralmente, as vantagens economicas conseguidas pela aplicação dos resultados obtidos, são o fato que torna importante esta metodologia. Tais funções permitem conhecimento mais amplo dos fenômenos biológicos que ocorrem na adubação e, consequentemente, conduzem a maior racionalidade no seu emprego técnico (Vieira et al., 1971).

Box \& Wilson (1951) introduziram os modelos de superfície de resposta para interpretar experimentos industriais. Eles são, hoje, empregados nos esquemas fatoriais $3^{k}$ dos ensaios agrícolas. Segundo esses autores, a resposta $\eta$, em qualquer ponto $\left(x_{1}, \ldots x_{t}, \ldots x_{k}\right)$ no espaço dos fatores, pode ser representada por uma equação de regressão da forma

$$
\eta=\beta_{0}+\beta_{1} x_{1}+\beta_{2} x_{2}+\ldots+\beta_{11} x_{1}^{2}+\ldots+\beta_{12} x_{1} x_{2}+\ldots+\beta_{111} x_{1}^{3}+\ldots
$$

No espaço $\Re^{k}$ dos $\mathbf{k}$ fatores existe uma região fechada $\mathcal{R}$, limitada pelo próprio experimento, chamada região experimental. O problema consiste em achar um ponto $\left(x_{1}^{0}, \ldots x_{t}^{0}, \ldots x_{k}^{0}\right)$ da região $\mathcal{R}$ no qual $\eta$ seja um mínimo ou um máximo, dependendo do caso estudado. Segundo Vieira et al. (1971), através das funções de produção é possível determinar os níveis dos fatores que conduzam à mais alta receita líquida ou à máxima taxa de rendimento sobre o capital aplicado.

Embora isso tudo seja possível, parece existir consenso no sentido de desenvolver experimentos orientados preferencialmente a estas opções, isto é, à maximização da receita ou da taxa de rendimento do capital investido, pois é extremamente escassa a bibliografia referente à otimização do custo do produto. Talvez o 
paradigma dominante destes últimos tempos na atividade agrícola tenha sido aquele que propendia à maximização dos benefícios em dinheiro e, neste assunto, inscrevia-se o conceito de uso racional do adubo.

Hoje, produtores agropecuários, em geral, encontram-se competindo pela colocação dos seus produtos no mercado, numa situação onde é, frequentemente, a economia internacional que determina os preços de venda. Isso pode ocasionar complicações na venda do produto ou mesmo a impossibilidade de vendê-lo a um preço razoável. A idéia de produzir minimizando os custos de produção aparece como alternativa válida, quando existe a necessidade de continuar produzindo.

É com esse objetivo que esta pesquisa foi desenvolvida. Neste trabalho, gera-se uma função $\mathbf{G}$ a duas e, posteriormente, a três variáveis, que exprime a quantidade de produto agrícola por unidade de moeda investida. G é função de $\eta$, aproximada mediante a aplicação de um modelo de superfície de resposta e de uma função linear dos custos da lavoura, tanto fixos como variáveis.

Por exemplo, seja um experimento fatorial de adubação com dois fatores em très níveis, $\mathrm{N}$ e $\mathrm{P}_{2} \mathrm{O}_{5}$, por exemplo. A superfície de resposta polinomial (de segundo grau) ajustada é da forma:

$$
Y=a_{00}+a_{01} N+a_{02} N^{2}+a_{10} P+a_{20} P^{2}+a_{11} N P+\varepsilon .
$$

Correspondentemente, a função linear de custo de adubação será:

$$
D=m+t_{1} N+t_{2} P,
$$

onde $\mathbf{m}$ denota a despesa fixa da instalação da cultura e $t_{i}(i=1,2)$ indica o preço do nutriente $\mathbf{i}$.

Define-se a função $G(N, P)$ como o quociente:

$$
G(N, P)=\frac{a_{00}+a_{01} N+a_{02} N^{2}+a_{10} P+a_{20} P^{2}+a_{11} N P}{m+t_{1} N+t_{2} P} .
$$


As derivadas parciais de $G(N, P)$ são as seguintes

$$
\left\{\begin{array}{l}
\frac{\partial G}{\partial N}=\frac{\left(a_{01}+2 N a_{02}+P a_{11}\right)\left(m+t_{1} N+t_{2} P\right)-\left(a_{00}+a 01 N+a_{02} N^{2}+a_{10} P+a_{20} P^{2}+a_{11} N P\right) t_{1}}{\left(m+t_{1} N+t_{2} P\right)^{2}} \\
\frac{\partial G}{\partial P}=\frac{\left(a_{10}+2 P a_{20}+N a_{11}\right)\left(m+t_{1} N+t_{2} P\right)-\left(a_{00}+a 01 N+a_{02} N^{2}+a_{10} P+a_{20} P^{2}+a_{11} N P\right) t_{2}}{\left(m+t_{1} N+t_{2} P\right)^{2}}
\end{array}\right.
$$

Igualando a 0 e simplificando as expressões, chega-se ao seguinte sistema de equações:

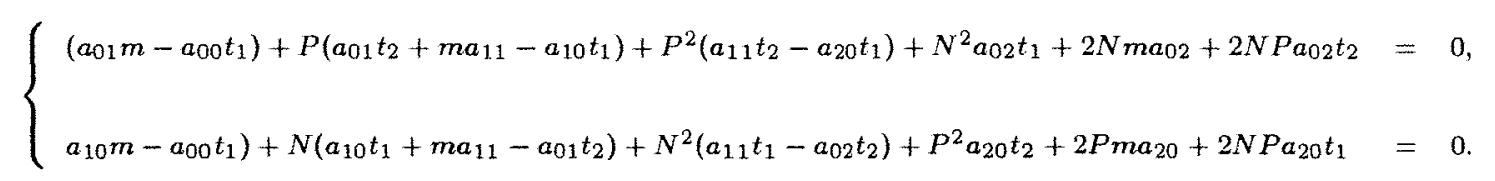

A eliminação de uma dessas incógnitas, $\mathrm{P}$ por exemplo, conduz a uma equação algébrica de quarto grau, no caso geral (Rey Pastor et al., 1969). Como se sabe, equações deste tipo têm solução algébrica complicadíssima, sendo portanto, pouco utilizadas no estudo de problemas objetivos. Aproxima-se, portanto, um modelo quadrático de superfície de resposta à função $G$ e procura-se o màximo mediante iterações sucessivas. O processo iterativo é justificado pelo interesse de que essa aproximação seja suficientemente exata para estimar o ponto de máximo com amplitude de variação menor que um determinado valor $\delta$, fixado arbitrariamente. Esse método será implementado com base no PROC RSREG do aplicativo SAS, criando malhas de pontos esperados, da função $G(N, P)$ e obtendo estimativas das coordenadas do ponto de máximo para malhas cada vez menores, até satisfazer a um critério de convergência. Se houver ponto de sela ou de mínimo, ou, ainda, se o máximo ocorrer fora da região experimental, busca-se o máximo absoluto da função, na fronteira dessa região. O ponto achado fornece a máxima quantidade de produto obtido por unidade de moeda investida. 
É interessante salientar que, embora seja utilizado neste trabalho um exemplo baseado na adubação de cana-de-açúcar, a técnica apresentada pode ser aplicada a qualquer cultivo agrícola ou florestal. Requer-se somente um grupo de experimentos fatoriais (ou um experimento com número suficiente de repeticões) com pelo menos dois fatores em três ou mais níveis. 


\section{REVISÃO DE LITERATURA}

Dado que o uso de polinômios no ajuste de equações de regressão é uma técnica bastante antiga, não há interesse, neste trabalho, de justificar seu uso salientando, porém, que os polinômios de segundo grau são usados na agricultura há muito tempo e com muitas vantagens (Pimentel-Gomes \& Conagin, 1987). A seguir, apresenta-se uma sucinta descrição dos avanços nesta área da Estatística Experimental, estritamente relacionados com este trabalho.

Foi durante as guerras mundiais que esta área experimentou o maior desenvolvimento. Já em 1941, Crowther \& Yates (citados por Heady \& Dillon, 1961), afirmam que as conclusões finais sobre a resposta a fertilizantes devem basear-se numa série de experimentos, conduzidos em diferentes anos, com diferentes culturas e em condições variadas de manejo e tipo de solo. Numerosos trabalhos subsequentes confirmam esta recomendação.

Um estudo detalhado sobre as funções de produção encontra-se em Heady \& Dillon (1961). Aspectos históricos, teoria, métodos e exemplos de interesse prático são apresentados de forma clara, para quem conhece conceitos básicos de economia e algumas técnicas estatísticas.

A técnica de superfície de resposta é hoje bastante utilizada nos ensaios de adubação com pelo menos dois nutrientes. É interessante salientar que a 
metodologia de superfície de resposta, apresentada nos trabalhos de Box \& Wilson (1951), Box (1954) e Box \& Hunter (1957), foi desenvolvida para tratar problemas da área industrial, onde muitas fontes de variação podem ser controladas e, consequentemente, o coeficiente de variação da variável resposta é baixo. Porém, isto não acontece na experimentação agronômica, onde pouco pode ser efetivamente controlado. Pimentel-Gomes \& Conagin (1987) tratam este problema em detalhe, desaconselhando o uso de delineamentos experimentais do tipo central composto na experimentação agronômica.

Um exemplo que utiliza a técnica de superfície de resposta para obtenção do ótimo econômico na utilização de fertilizantes é apresentado no trabalho de Heady \& Dillon (1961). Nele, são resumidos ressultados de estudos começados em 1952 , utilizando um ou mais dos nutrientes $\mathrm{N}, \mathrm{K}_{2} \mathrm{O}$ e $\mathrm{P}_{2} \mathrm{O}_{5}$. Os autores ajustam quatro tipos de equações através da técnica de superfície de resposta, trabalhando com os nutrientes $\mathrm{Ne} \mathrm{P}_{2} \mathrm{O}_{5}$. As funções são as de Cobb-Douglas e três formas polinomiais gerais (quadrática, raiz quadrada e potência $\frac{3}{2}$ ).

Uma aproximação a três variáveis para o problema da adubação pode ser vista em Campos (1967) que analisou um grupo de 50 experimentos fatoriais $3^{3}$ em milho. A metodologia de superfície de resposta é utilizada para fazer inferências sobre os nutrientes $\mathrm{N}, \mathrm{K}_{2} \mathrm{O}$ e $\mathrm{P}_{2} \mathrm{O}_{5}$. Analisa vários trabalhos onde o baixo número de observações e o mau ajuste do modelo levam a problemas na estimação dos parâmetros. Definindo níveis ótimos como aqueles que conduzem ao rendimento financeiro líquido máximo do emprendimento, fica claro o enfoque do estudo desenvolvido pelo autor. Ele demonstra que as estimativas dos parâmetros da superfície de resposta possuem amplos intervalos de confiança sendo, portanto, de pouca precisão. Concluiu, também, que a grande maioria dos experimentos estudados apresentam ponto de sela quando analisados individualmente mas, à medida que se faz o agrupamento dos ensaios, existe maior tendência ao aparecimento de ponto de máximo. 
A análise de 50 experimentos demonstrou que a técnica de superfície de resposta deve ser aplicada com cuidado nos casos individuais, pois não é raro ocorrer ponto de mínimo onde deveria aparecer ponto de máximo, doses negativas ou absurdamente grandes de nutrientes ou, o mais comum, pontos de sela que dificultam a recomendação de dose ótima. Estudou detalhadamente a dose econômica de nutrientes, demonstrando que esta é de grande sensibilidade e que apresenta, em geral, amplitude de variação inaceitável no campo agronômico. O autor recomenda, também, que a aplicação da técnica de superfície de resposta nos ensaios fatoriais $3^{3}$ de adubação seja aplicada somente a grupos de experimentos de boa precisão. No entanto, aqui também é desaconselhado o uso de delineamentos do tipo "central composto", já que o esquema fatorial completo de tratamentos demonstrou ser melhor do que o primeiro, pois as estimativas dos parâmetros possuiam menor variância.

Um enfoque econômico ao problema da adubação encontra-se em Zagatto \& Pimentel-Gomes (1960), baseado na lei das proporções variáveis, dos rendimentos decrescentes, dos acréscimos não proporcionais ou ainda na lei de Mitscherlich. A importância desse trabalho, embora com uma só variável, concentra-se no estudo em condições de capital escasso, onde os autores demonstram que a quantidade ótima de nutriente $\mathrm{X}_{0}$ é a que torna máxima a relação entre o rendimento líquido e o custo bruto, não sendo tão importante a maximização do rendimento do capital investido. Desenvolvimentos mais detalhados sobre dose ótima, baseados na lei de Mitscherlich, encontram-se em Pimentel-Gomes \& Abreu (1959); Pimentel-Gomes (1961) e d'Aulísio (1973).

Jorge \& Conagin (1977), baseados em simulações de 60 experimentos tanto individuais como reunidos em grupos de tamanhos crescentes, concluem que:

- existe um aumento na porcentagem de pontos de máximo à medida que se aumenta o número de experimentos analisados; 
- o ponto de máximo para a função de receita líquida tende a aparecer mais frequentemente dentro da região experimental quanto maior é o número de experimentos utilizado.

O único trabalho encontrado que se refere especificamente ao custo mínimo unitário de produção é o de Pimentel-Gomes \& Garcia (1995), que analisaram o problema da dose de adubo que permite a produção de madeira de custo mínimo, em função de dados experimentais, trabalhando com Eucalyptus grandis. Usaram uma equação polinomial de segundo grau

$$
V(x)=a+b x+c x^{2}
$$

onde $\mathrm{x}$ é a dose do fertilizante, em $\mathrm{kg} / \mathrm{ha}$, e $\mathrm{V}$ é o volume, que se supõe em st/ha. Utilizaram, além disso, uma função linear de custos

$$
D=m+t x
$$

onde $\mathbf{t}$ é o preço do quilograma de fertilizante e $\mathbf{m}$ refere-se a despesas fixas do povoamento. Trabalhando com essas funções, criaram a função $G(x)$ que exprime a quantidade de madeira obtida por dólar investido. O objetivo do trabalho foi obter o nível ótimo de adubação $\mathrm{X}_{0}>0$ que forneça a produção máxima de madeira por dólar. Pelas características da metodologia usada, valores negativos de $\mathrm{X}_{0}$ indicam que não convém adubar.

Como conclusão, o polinômio de segundo grau é uma ferramenta muito utilizada no estudo dos ensaios de adubação, mas seu uso requer certos cuidados a fim de não cair no abuso. Estudos relacionados com a estimação por intervalo do ponto crítico de uma equação quadrática encontram-se em d'Aulí sio,(1976) e Gomes (1989). Uma análise detalhada sobre os problemas e possíveis soluções para o uso de superfícies de resposta em ensaios de adubação encontra-se em Zagatto \& Pimentel-Gomes (1967). Sucintamente, os problemas podem assim ser resumidos: 
- a equação para a receita líquida pode não ter máximo, mas sim mínimo ou ponto de sela. Isto é particularmente frequente na análise de experimentos isolados. Pode ocorrer o aparecimento de valores absurdos nessas análises;

- o ajustamento pode ser pouco satisfatório;

- doses ótimas, quando ocorrem, podem ter erro padrão muito elevado e, portanto, pouca precisão.

- "As superfícies de resposta obtidas de ensaios em condições similares são frequentemente bem distintas. Isto pode mesmo ocorrer até em um mesmo ensaio quando se muda o método de amostragem" (Tejeda e Hoffnarr \& Johnson, citados por Zagatto \& Pimentel-Gomes, 1967);

- não é raro ocorrer que, em análises individuais, nenhum ou quase nenhum dos coeficientes do polinômio estimado seja significativo, o que, evidentemente, torna o método inseguro do ponto de vista prático.

Embora esses problemas sejam impossiveis de eliminar, pode-se minimizar seus efeitos indesejáveis. Para contornar essas dificuldades os autores recomendam:

- usar sempre grupos de ensaios numerosos ou ensaios isolados com muitas repetições e boa precisão;

- não confiar em doses ótimas obtidas a partir de polinômios de segundo grau, sem a prévia verificação de que os coeficientes dos termos de segundo grau são significativamente diferentes de zero;

- verificar sempre se os valores obtidos correspondem realmente a um ponto de máximo; 
- construir os intervalos de confiança para as doses ótimas encontradas, para poder julgar o seu real valor prático.

Em favor do uso do polinômio de segundo grau, Pimentel-Gomes \& Conagin (1987) comparando-o com o polinômio com raiz quadrada, a função de Baule (generalização da lei de Mitscherlich) e a função de Cobb-Douglas generalizada, concluem que o primeiro é recomendável já que:

- "Seu uso é muito mais fácil.

- Nos casos mais comuns não é difícil obter estimadores independentes para os coeficientes da equação.

- Complicações, tais como o aparecimento de pontos de sela, são menos comuns nos polinômios do segundo grau, do que nos polinômios com raiz quadrada" (Pimentel-Gomes \& Conagin, 1987, p. 28). 


\section{MATERIAL E MÉTODOS}

\subsection{Material}

Os dados utilizados foram obtidos do trabalho realizado por Malavolta et al. (1963), sobre diagnose foliar na cana-de-açúcar. Nele analisam-se 40 ensaios fatoriais $3^{3}$ de adubação, cada um com uma só repetição dos 27 tratamentos, em blocos de 9 parcelas e com confundimento de dois graus de liberdade da interação tripla com os blocos, conforme a estrutura de tratamentos conhecida como "grupo W", apresentada por Yates no ano de 1937.

Os experimentos foram instalados em 1958, quando se usava ainda como adubo nitrogenado o Salitre-do-Chile. Portanto, realizou-se a conversão desses valores para níveis de nitrogênio provenientes de uréia. As doses de adubo correspondiam a $0 ; 60$ e $120 \mathrm{~kg} / \mathrm{ha}$ de $\mathbf{N}$. Analogamente, realizou-se a conversão de hiperfosfato (hoje sem utilização) para superfosfato simples, correspondente a doses de 0;75 e $150 \mathrm{~kg}$ de $\mathrm{P}_{2} \mathrm{O}_{5}$ por hectare. Estas mesmas quantidades foram utilizadas também para o potássio $\left(\mathrm{K}_{2} \mathrm{O}\right)$, aplicado na forma de cloreto.

Os 40 experimentos mencionados foram instalados em diferentes localidades do Estado de São Paulo. Os autores dividem o grupo em três subgrupos, segundo o tipo de solo: Nitossolo (antes terra roxa misturada), terra roxa legítima e solos de tipos diversos. Para este estudo foi escolhido o primeiro subgrupo constituido 
por 15 experimentos.

Realizadas as análises da variância individuais pelos autores desse trabalho, um experimento foi excluído da análise conjunta devido ao excessivo valor do seu quadrado médio do resíduo. Na tabela seguinte, apresentam-se os quadrados médios residuais para cada experimento.

Tabela 1: Quadrados Médios Residuais

\begin{tabular}{|c|c|c|c|c|c|}
\hline & Q.M. & & Q.M. & & Q.M. \\
\hline Ensaio & Residual & Ensaio & Residual & Ensaio & Residual \\
\hline 1 & 155,85 & 12 & 97,85 & 30 & 130,81 \\
\hline 2 & 53,76 & 18 & 126,76 & 33 & 236,27 \\
\hline 3 & 93,26 & 21 & 189,55 & 34 & 175,10 \\
\hline 4 & 140,30 & 24 & $1.069,27$ & 37 & 233,57 \\
\hline 11 & 120,42 & 27 & 185,39 & 38 & 250,69 \\
\hline
\end{tabular}

Como este estudo está baseado na técnica da análise da variância, o ensaio número 24 foi excluído, devido a discrepância entre este Q. M. e os demais (a razão entre o maior e o menor é de aproximadamente 20). Eliminado este experimento, a razão de variâncias cai para $\frac{250,69}{53,76}=4,7$, valor este que, sem chegar a ser muito grande, justifica o uso da técnica do resíduo específico (Cochran \& Cox, 1957) para melhorar a estimação dos efeitos de interesse. Os dados se apresentam no Apêndice 1. Os preços dos nutrientes, foram os correspondentes ao mês de agosto de 1997.

Tabela 2: Preços dos adubos

\begin{tabular}{|l|l|l|}
\hline Adubo & Preço & Nutriente (\%) \\
\hline Cloreto de Potássio (t) & $\mathrm{R} \$ 357,39$ & $60\left(\mathrm{~K}_{2} \mathrm{O}\right)$ \\
Superfosfato simples (t) & $\mathrm{R} \$ 227,80$ & $20\left(\mathrm{P}_{2} \mathrm{O}_{5}\right)$ \\
Uréia (t) & $\mathrm{R} \$ 444,48$ & $45(\mathrm{~N})$ \\
\hline
\end{tabular}


Os níveis utilizados nas análises seguem a codificação imposta aos fatores para o ajuste da superfície de resposta:

$$
N^{*}=\frac{N}{60} \quad P^{*}=\frac{P}{75} \quad K^{*}=\frac{K}{75} .
$$

Como exemplo, a dose 1 do nitrogênio corresponde a $60 \mathrm{~kg}$ deste nutriente por hectare e equivale a $130 \mathrm{~kg} / \mathrm{ha}$ de uréia. Para simplificar a notação, os nutrientes $\mathrm{P}_{2} \mathrm{O}_{5}$ e $\mathrm{K}_{2} \mathrm{O}$ serão indicados com $\mathrm{P}$ e K respectivamente.

Todas as análises estatísticas foram realizadas com o aplicativo SAS, versão 6.08 para Windows (1989), enquanto que os gráficos foram realizados com o STATISTICA, versão 5.1 para Windows (1997).

\subsection{Métodos}

A metodologia utilizada está baseada na análise da variância e posterior ajuste de um modelo empírico de superfície de resposta.

Primeiramente, ajustou-se o modelo:

$$
\begin{aligned}
y_{i j k l m}= & \mu+\alpha_{i}+\beta \alpha_{(i) j}+N_{k}+P_{l}+K_{m}+ \\
& +(N P)_{k l}+(N K)_{k m}+(P K)_{l m}+(N P K)_{k l m}+ \\
& +(\alpha N)_{i k}+(\alpha P)_{i l}+(\alpha K)_{i m}+ \\
& +(\alpha N P)_{i k l}+(\alpha N K)_{i k m}+(\alpha P K)_{i l m}+\varepsilon_{i j k l m}
\end{aligned}
$$

onde:

$y_{i j k l m}$ denota a produtividade obtida por real, observada no experimento $i$, bloco $j$ para a $k l m$ - ésima combinação dos fatores $\mathrm{N}, \mathrm{P}$ e K respectivamente; $\mu$ denota a média geral; 
$\alpha_{i}$ denota o efeito do experimento $i$, (considerado aleatório);

$\beta \alpha_{(i) j}$ denota o efeito dos blocos dentro do experimento $i$ (considerado aleatório);

$N_{k}$ denota o efeito de fator nitrogênio (considerado fixo);

$P_{l}$ denota o efeito do fator fósforo (considerado fixo);

$K_{m}$ denota o efeito de fator potássio (considerado fixo);

$(\mathrm{NP})_{k l} ;(\mathrm{NK})_{k m}$ e $(\mathrm{PK})_{l m}$ indicam os efeitos das respectivas interações duplas para

$\mathrm{N}, \mathrm{P}$ e K (considerados fixos);

$(\mathrm{NPK})_{k l m}$ indica o efeito da interação tripla para os efeitos $\mathrm{N}, \mathrm{P}$ e K (considerado fixo);

$(\alpha \mathrm{N})_{i k} ;(\alpha \mathrm{P})_{i l}$ e $(\alpha \mathrm{K})_{i m}$ denotam os efeitos das interaçóes dos fatores com os locais dos experimentos (considerados aleatórios);

$(\alpha \mathrm{NP})_{i k l} ;(\alpha \mathrm{NK})_{i k m}$ e $(\alpha \mathrm{PK})_{i l m}$ denotam os efeitos das interações entre as interações de primeira ordem dos fatores $\mathrm{N}, \mathrm{Pe} \mathrm{K}$, com os experimentos (considerados aleatórios).

O teste $\mathbf{F}$ é realizado utilizando os denominadores apropriados, segundo determinam as esperanças dos respectivos quadrados médios. É utilizada a técnica do resíduo específico, apresentada por Cochran \& Cox (1957), para melhorar a estimação da estatística $\mathbf{F}$ para cada coeficiente do polinômio. Um polinômio de segundo grau é a justado para representar aproximadamente a função $G(N, P, K)$. Foi realizado, também para este último, um teste de falta de ajuste. Os coeficientes do polinômio de segundo grau foram estimados pelo procedimento REG, do aplicativo SAS. Uma vez estimado o polinômio, faz-se a análise canônica da superfície, para verificar se existe máximo para a função $G(N, P, K)$. Analogamente, a minimização da inversa de $G(N, P, K)$ determinaria o custo mínimo de produção por unidade de produto.

Quando o máximo da função encontra-se fora da região experimental analisada, ou quando a função $\mathrm{G}(\mathrm{N}, \mathrm{P}, \mathrm{K})$ tem ponto de sela ou de mínimo, utiliza-se 
o método de steepest ascent (Box \& Wilson, 1951). Esta metodologia está implementada no procedimento RSREG do SAS, através da opção ridge. O único inconveniente a destacar é que o aplicativo não reconhece os limites da região experimental, devendo o pesquisador, em consequencia, orientar o programa de maneira intuitiva, verificando, a cada passo, as coordenadas dos pontos apresentados e modificando, quando necessário, a direção de procura.

Para facilitar a abordagem do problema, iniciou-se o trabalho com apenas duas variáveis. Para isso, foram escolhidos os fatores com maior valor da estatística $\mathbf{F}$, dentre os três possíveis na análise de variância. A seguir, fez-se o ajustamento de uma função polinomial de segunda ordem para estimar os efeitos linear e quadrático dos fatores analisados. Foi realizado o estudo do comportamento da função $\mathrm{G}$ assim obtida. 


\section{RESULTADOS E DISCUSSÃO}

\subsection{Análise da Variância}

Em função do modelo matemático proposto, realizou-se a análise da variância do conjunto dos 14 experimentos. O ajuste do modelo geral, com 299 parâmetros, apresenta-se no quadro seguinte, obtido através do procedimento GLM do SAS.

Tabela 3: Modelo geral: análise da variância.

\begin{tabular}{lrrccc}
\hline \multicolumn{1}{c}{ Causa de } & \multicolumn{2}{c}{ Somas de } & Quadrados & \\
Variação & G.L. & Quadrados & Médios & F & Probabilidade \\
\hline Modelo & 299 & $256.241,737$ & 856,996 & 5,45 & 0,0001 \\
Resíduo & 78 & $12.258,368$ & 157,159 & & \\
\hline Total corrigido & 377 & $268.500,105$ & & &
\end{tabular}

Conclui-se, portanto, que o modelo em estudo ajusta-se bem aos dados. Isto é esperado dada a complexidade do modelo ajustado, pois quase $80 \%$ dos graus de liberdade disponíveis são utilizados para estimar parâmetros. Mas, nas análises especificas para os estudos com duas e três variáveis, as somas de quadrados associadas a efeitos que não são interessantes aos fins deste trabalho, são acrescentadas ao resíduo. O coeficiente de variação do experimento foi de $10,2 \%$, considerado muito bom tendo em vista que os dados vêm de 14 locais diferentes distantes entre si. 


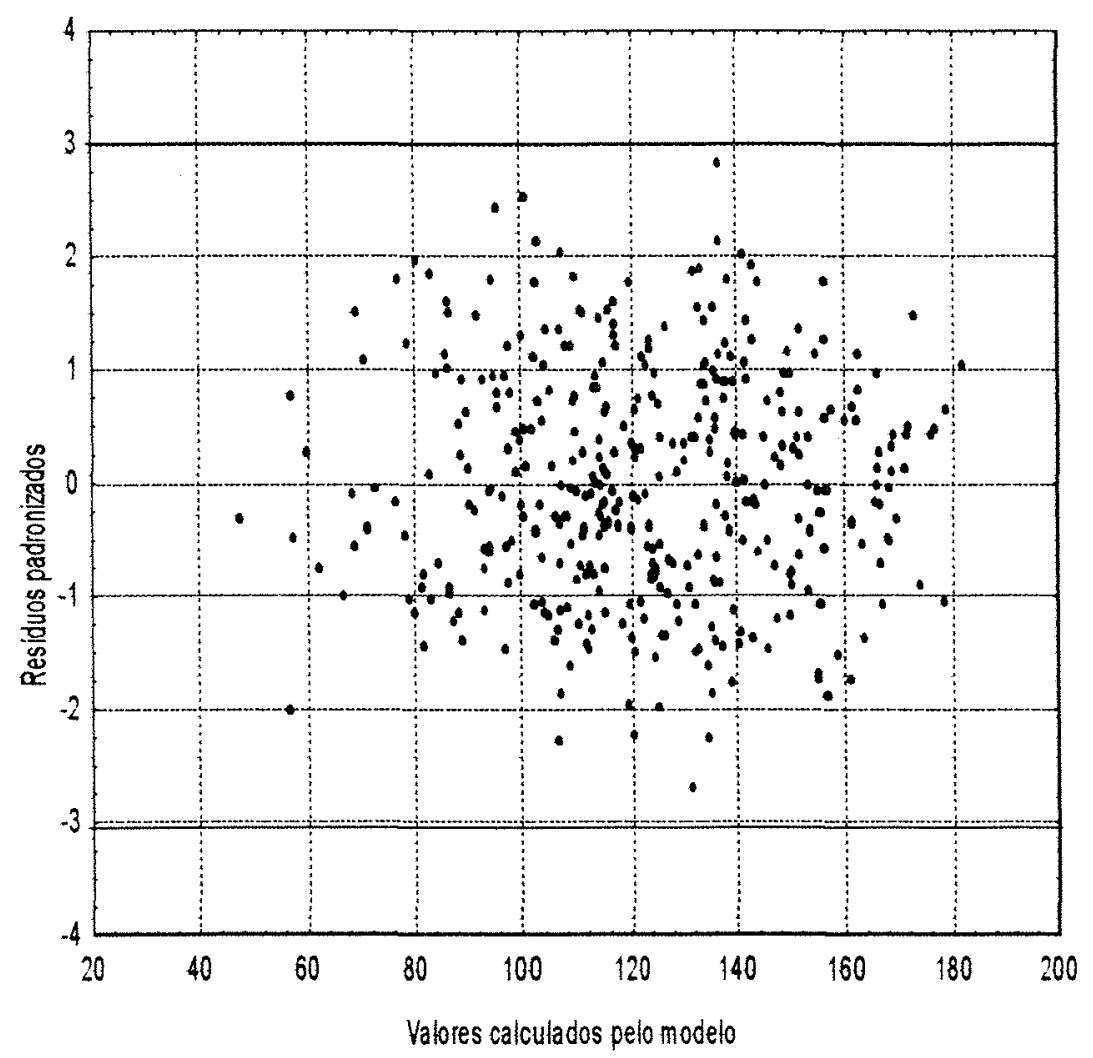

Figura 1: Modelo geral: dispersão dos resíduos padronizados.

Uma idéia da dispersão dos resíduos padronizados em função dos valores calculados pelo modelo aparece na Figura 1 e permite admitir que não existem problemas quanto às pressuposições da análise da variância. Não se observa a existência de valores extranhos (outliers) ou de um padrão definido de dispersão que sugira uma possível heterogeneidade de variâncias ou correlação serial entre os resíduos. Em função disso, considera-se que as pressuposições da análise da variância são respeitadas. Deve-se salientar que, para contornar um possível efeito indesejável por causa das variâncias dos experimentos individuais, a técnica do resíduo específico é utilizada.

Verificadas as pressuposições da análise da variância, o modelo geral é desdobrado segundo os efeitos de interesse, isto é experimentos, restrições à casua- 
lização (o efeito dos blocos dentro de experimentos), efeitos principais (N, $\mathrm{P}$ e K) e interações $(N \times P, N \times K, P \times K$ e $N \times P \times K)$, segundo o modelo especificado acima. Dado que existe confundimento de dois graus de liberdade da interaç̧ão $\mathrm{N} \times \mathrm{P} \times \mathrm{K}$ com os blocos dentro de cada experimento, é utilizada a soma de quadrados sequencial (Tipo I do SAS), ajustando experimentos ignorando outros fatores, depois o efeito blocos(experimentos) e, posteriormente, os fatores de interesse propriamente ditos, já que essa sequência tem sentido, pois a interação $\mathrm{N} \times \mathrm{P} \times \mathrm{K}$ estará ajustada para os blocos. A seguir, apresentam-se as esperanças dos quadrados médios para este estudo, determinadas pelo SAS, sempre considerando a incorporação sequencial dos efeitos no modelo.

Efeitos de interesse

$$
\begin{aligned}
\operatorname{EXPER}(\mathbf{E})= & \sigma^{2}+3 \sigma_{E \times P \times K}^{2}+3 \sigma_{E \times N \times P}^{2}+3 \sigma_{E \times N \times K}^{2} \\
& +9 \sigma_{E \times P}^{2}+9 \sigma_{E \times K}^{2}+9 \sigma_{E \times N}^{2}+9 \sigma_{B L O C O(E)}^{2} \\
& +27 \sigma_{E}^{2} \\
\mathbf{N}= & \sigma^{2}+3 \sigma_{E \times N \times P}^{2}+3 \sigma_{E \times N \times K}^{2}+9 \sigma_{E \times N}^{2} \\
& +Q_{N, N \times P, N \times K, N \times P \times K} \\
\mathbf{P}= & \sigma^{2}+3 \sigma_{E \times P \times K}^{2}+3 \sigma_{E \times N \times P}^{2}+9 \sigma_{E \times P}^{2} \\
& +Q_{(P, N \times P, P \times K, N \times P \times K)} \\
\mathbf{K}= & \sigma^{2}+3 \sigma_{E \times P \times K}^{2}+3 \sigma_{E \times N \times K}^{2}+9 \sigma_{E \times K}^{2} \\
& +Q_{(K, N \times K, P \times K, N \times P \times K)} \\
\mathbf{N} \times \mathbf{P}= & \sigma^{2}+3 \sigma_{E \times N \times P}^{2}+Q_{(N \times P, N \times P \times K)} \\
\mathbf{N} \times \mathbf{K}= & \sigma^{2}+3 \sigma_{E \times N \times K}^{2}+Q_{(N \times K, N \times P \times K)} \\
\mathbf{P} \times \mathbf{K}= & \sigma^{2}+3 \sigma_{E \times P \times K}^{2}+Q_{(P \times K, N \times P \times K)} \\
\mathbf{N} \times \mathbf{P} \times \mathbf{K}= & \sigma^{2}+Q_{(N \times P \times K)}
\end{aligned}
$$


Respectivos denominadores para o teste de $\mathrm{F}$

$$
\begin{aligned}
\mathbf{E}= & \sigma^{2}+3 \sigma_{E \times P \times K}^{2}+3 \sigma_{E \times N \times P}^{2}+3 \sigma_{E \times N \times K}^{2} \\
& +9 \sigma_{E \times P}^{2}+9 \sigma_{E \times K}^{2}+9 \sigma_{E \times N}^{2}+9 \sigma_{B L O C O(E)}^{2} \\
\mathbf{E} \times \mathbf{N}= & \sigma^{2}+3 \sigma_{E \times N \times P}^{2}+3 \sigma_{E \times N \times K}^{2}+9 \sigma_{E \times N}^{2} \\
\mathbf{E} \times \mathbf{P}= & \sigma^{2}+3 \sigma_{E \times P \times K}^{2}+3 \sigma_{E \times N \times P}^{2}+9 \sigma_{E \times P}^{2} \\
\mathbf{E} \times \mathbf{K}= & \sigma^{2}+3 \sigma_{E \times P \times K}^{2}+3 \sigma_{E \times N \times K}^{2}+9 \sigma_{E \times K}^{2} \\
\mathbf{E} \times \mathbf{N} \times \mathbf{K}= & \sigma^{2}+3 \sigma_{E \times N \times K}^{2} \\
\mathbf{E} \times \mathbf{N} \times \mathbf{P}= & \sigma^{2}+3 \sigma_{E \times N \times P}^{2} \\
\mathbf{E} \times \mathbf{P} \times \mathbf{K}= & \sigma^{2}+3 \sigma_{E \times P \times K}^{2}
\end{aligned}
$$

Aqui, o símbolo $\sigma^{2}$ denota componente de variância e o $\mathrm{Q}$ indica forma quadrática nos fatores envolvidos, segundo a notação usada pelo SAS. Deve-se salientar que, nessa forma quadrática, não são impostas restrições, portanto ela não corresponde à hipótese testada.

A tabela da análise de variância apresenta-se a seguir. Todos os efeitos foram testados respeitando as esperanças dos quadrados médios apresentados e indica-se por dois asteriscos a significância ao nível de $1 \%$ de probabilidade. 
Tabela 4: Desdobramento do modelo geral: análise da variância.

\begin{tabular}{|c|c|c|c|c|c|}
\hline $\begin{array}{l}\text { Causa de } \\
\text { Variação }\end{array}$ & G.L. & $\begin{array}{l}\text { Somas de } \\
\text { Quadrados }\end{array}$ & $\begin{array}{c}\text { Quadrado } \\
\text { Médios }\end{array}$ & $\mathbf{F}$ & Probabilidade \\
\hline EXPERIMENTOS (E) & 13 & $157.587,27$ & $12.122,10$ & 16,46 & $0,0001^{* *}$ \\
\hline $\operatorname{BLOCOS}(\mathrm{E})$ & 28 & $10.603,32$ & 378,69 & & \\
\hline $\mathrm{N}$ & 2 & $12.634,96$ & $6.317,48$ & 16,23 & $0,0001^{* *}$ \\
\hline $\mathrm{P}$ & 2 & $2.675,91$ & $1.337,96$ & 7,37 & $0,0029^{* *}$ \\
\hline K & 2 & $22.796,08$ & $11.398,04$ & 47,25 & $0,0001^{* *}$ \\
\hline $\mathrm{N} \times \mathrm{P}$ & 4 & 239,82 & 59,95 & 0,31 & $0,8720 \mathrm{~ns}$ \\
\hline $\mathrm{N} \times \mathrm{K}$ & 4 & $1.543,68$ & 385,92 & 2,23 & $0,0770 \mathrm{~ns}$ \\
\hline $\mathrm{P} \times \mathrm{K}$ & 4 & 573,65 & 143,41 & 1,67 & $0,1700 \mathrm{~ns}$ \\
\hline $\mathrm{N} \times \mathrm{P} \times \mathrm{K}$ (não conf.) & 6 & $2.849,34$ & 474,89 & 3,02 & $0,0100^{* *}$ \\
\hline$E \times N$ & 26 & $10.118,35$ & 389,17 & & \\
\hline $\mathrm{E} \times \mathrm{P}$ & 26 & $4.721,96$ & 181,61 & & \\
\hline $\mathrm{E} \times \mathrm{K}$ & 26 & $6.271,47$ & 241,21 & & \\
\hline $\mathrm{E} \times \mathrm{N} \times \mathrm{P}$ & 52 & $10.186,11$ & 195,89 & & \\
\hline $\mathrm{E} \times \mathrm{N} \times \mathrm{K}$ & 52 & $8.978,34$ & 172,66 & & \\
\hline $\mathrm{E} \times \mathrm{P} \times \mathrm{K}$ & 52 & $4.461,46$ & 85,79 & & \\
\hline RESÍDUO & 78 & $12.258,37$ & 157,16 & & \\
\hline
\end{tabular}

\section{Interpretação}

Existe efeito significativo de locais e a utilização de blocos contribuiu na redução da soma de quadrados do resíduo, aumentando a eficiência do teste $\mathbf{F}$ para a interação tripla, que resultou significativa ao nível de probabilidade de $1 \%$. Foram detectados efeitos significativos dos fatores em estudo e existe evidência, ao nível de $8 \%$ de significância da interação $N \times K$, porém não foram comprovados efeitos das outras duas interações duplas. Consequentemente, iniciou-se o estudo com duas variáveis apenas, a fim de compreender o processo, utilizando as variáveis nitrogênio e potássio, já que os respectivos valores da estatística $\mathrm{F}$ são os maiores dos três possíveis. Além disso, o valor relativamente alto de $\mathrm{F}$ para a interação $\mathrm{N} \times \mathrm{K}$ 
$(\mathbf{F}=2,23)$ sugere ao pesquisador o estudo com maior detalhe do problema.

\subsection{Estudo com Duas Variáveis}

Para começar a pesquisa, deve-se procurar a existência de efeitos polinomiais associados aos fatores estudados, a fim de gerar a função de produtividade $\mathbf{Y}(\mathbf{N}, \mathbf{K})$. Dada a quantidade de experimentos utilizados neste trabalho, a técnica do resíduo específico pode ser utilizada para aumentar a sensibilidade do teste $\mathrm{F}$ para os efeitos de interesse. Uma desvantagem desta técnica é a diminuição dos graus de liberdade nos denominadores utilizados $\left(\mathrm{N}^{\prime} \times\right.$ Experimentos, $\mathrm{N}^{\prime \prime} \times$ Experimentos e assim por diante), mas com 14 experimentos analisados, essa desvantagem não dificulta a análise e os efeitos polinomiais de interesse são testados com maior eficiência, sem pôr em risco a precisão das estimativas dos Q.M. dos denominadores.

\subsubsection{Análise da Variância}

A análise de variância aparece na Tabela 5. O efeito quadrático do nitrogênio $\left(\mathrm{N}^{\prime \prime}\right)$ foi desconsiderado, pois não foi significativo num ajuste preliminar $(\mathbf{F}=0,87)$. Imediatamente abaixo de cada efeito de interesse, segue-se o resíduo específico do teste. 
Tabela 5: $\mathrm{Y}(\mathrm{N}, \mathrm{K})$ : análise da variância.

\begin{tabular}{lrrrcc}
\hline \multicolumn{1}{c}{ Causa de } & \multicolumn{2}{c}{ Somas de } & Quadrados & \\
\multicolumn{1}{c}{ Variação } & g.l. & Quadrados & \multicolumn{1}{c}{ Médios } & F & Probabilidade \\
\hline EXPERIMENTOS (E) & 13 & $157.587,270$ & $12.122,10$ & 16,46 & $0,0001^{* *}$ \\
BLOCOS(E) & 28 & $10.603,32$ & 378,69 & & \\
$\mathrm{~N}^{\prime}$ & 1 & $12.363,40$ & $12.363,40$ & 26,50 & $0,0002^{* *}$ \\
$\mathrm{E} \times \mathrm{N}^{\prime}$ & 13 & $6.064,79$ & 466,52 & & \\
$\mathrm{~K}^{\prime}$ & 1 & $20.153,62$ & $20.153,62$ & 55,02 & $0,0001^{* *}$ \\
$\mathrm{E} \times \mathrm{K}^{\prime}$ & 13 & $4.762,07$ & 366,31 & & \\
$\mathrm{~K}^{\prime \prime}$ & 1 & $2.642,46$ & $2.642,46$ & 22,76 & $0,0004^{* *}$ \\
$\mathrm{E} \times \mathrm{K}^{\prime \prime}$ & 13 & $1.509,40$ & 116,11 & & \\
$\mathrm{~N}^{\prime} \times \mathrm{K}^{\prime}$ & 1 & 996,21 & 996,21 & 4,44 & $0,0550 *$ \\
$\mathrm{E} \times \mathrm{N}^{\prime} \times \mathrm{K}^{\prime}$ & 13 & $2.913,26$ & 224,10 & & \\
$\mathrm{P}$ & 2 & $2.675,91$ & $1.337,96$ & 7,37 & $0,0029^{* *}$ \\
$\mathrm{~N} \times \mathrm{P}$ & 4 & 239,82 & 59,95 & 0,31 & $0,8720 \mathrm{~ns}$ \\
$\mathrm{P} \times \mathrm{K}$ & 4 & 573,65 & 143,41 & 1,67 & $0,1700 \mathrm{~ns}$ \\
$\mathrm{~N} \times \mathrm{P} \times \mathrm{K}$ (não conf.) & 6 & $2.849,34$ & 474,89 & 2,74 & $0,0100^{* *}$ \\
$\mathrm{E} \times \mathrm{P}$ & 26 & $4.721,96$ & 181,61 & & \\
$\mathrm{E} \times \mathrm{N} \times \mathrm{P}$ & 52 & $10.186,11$ & 195,89 & & \\
$\mathrm{E} \times \mathrm{P} \times \mathrm{K}$ & 52 & $4.461,46$ & 85,79 & & \\
$\mathrm{RESIDUO}$ & 134 & $23.196,04$ & 173,11 & & \\
\hline TOTAL CORRIGIDO & 377 & $268.500,10$ & & & \\
& & & & & \\
& & & &
\end{tabular}

\section{Interpretação}

Verifica-se, pois, a existência de efeitos lineares do nitrogênio e do potássio, mas só efeito quadrático deste último. Confere-se, também, que a interação $\mathrm{N}^{\prime} \times K^{\prime}$ é praticamente significativa ao nível de probabilidade de $5 \%$ e que este efeito estava mascarado pelas interações, correspondentes aos outros três graus de liberdade, no teste original.

Agrupando convenientemente as somas de quadrados obtidas na análise anterior, podem ser obtidas estatísticas $\mathbf{F}$ para testar hipóteses de interesse para o 
trabalho em relação à superficie ajustada. Na Tabela 6, testaram-se as seguintes hipóteses:

- o modelo de regressão polinomial de segundo grau se ajusta bem em todos os locais estudados?

- os desvios de regressão são significativos?

Tabela 6: Y(N, K): teste de falta de ajuste.

\begin{tabular}{lrrrrr}
\hline \multicolumn{1}{c}{ Causa de } & \multicolumn{2}{c}{ Somas de } & Quadrados & \\
\multicolumn{1}{c}{ Variação } & G.L. & Quadrados & Médios & \multicolumn{1}{c}{ F } & Probabilidade \\
\hline EXPERIMENTOS (E) & 13 & $157.587,27$ & $12.122,10$ & 16,46 & $0,0001^{* *}$ \\
BLOCOS(E) & 28 & $10.603,32$ & 378,69 & & \\
Outras Fontes & 146 & $25.708,25$ & & & \\
\hline REGRESSÃO & 4 & $36.155,69$ & $9.038,92$ & 24,76 & $0,0001^{* *}$ \\
REGRESSÃO $\times$ E & 52 & $15.296,52$ & 294,16 & & \\
\hline DESVIOS DE REG. & 4 & 819,03 & 204,75 & 1,05 & $0,332 \mathrm{~ns}$ \\
DESVIOS $\times$ E & 52 & $10.118,64$ & 194,59 & & \\
RESÍDUO & 78 & $12.258,36$ & 157,15 & & \\
\hline TOTAL CORRIGIDO & 377 & $268.500,10$ & & & \\
\hline
\end{tabular}

A expressão "Outras Fontes" corresponde a efeitos desinteressantes neste caso, isto é, os relacionados com o fator fósforo. Da leitura da tabela, concluise que, na primeira hipôtese, existe evidência altamente significativa para rejeitá-la e, contrariamente, no segundo caso, a hipótese de nulidade não é rejeitada. Fica demonstrado, pois, que a superfície de resposta utilizada representa bem o fenômeno. Uma análise visual do ajuste do modelo pode ser conseguida através de um gráfico de dispersão dos resíduos padronizados. Este gráfico apresenta-se na Figura 2 . O coeficiente de determinação é $\mathbf{R}^{2}=\mathbf{0 , 8 3}$, calculado pelo quociente $S Q$ Regressão por SQ Tratamentos. 


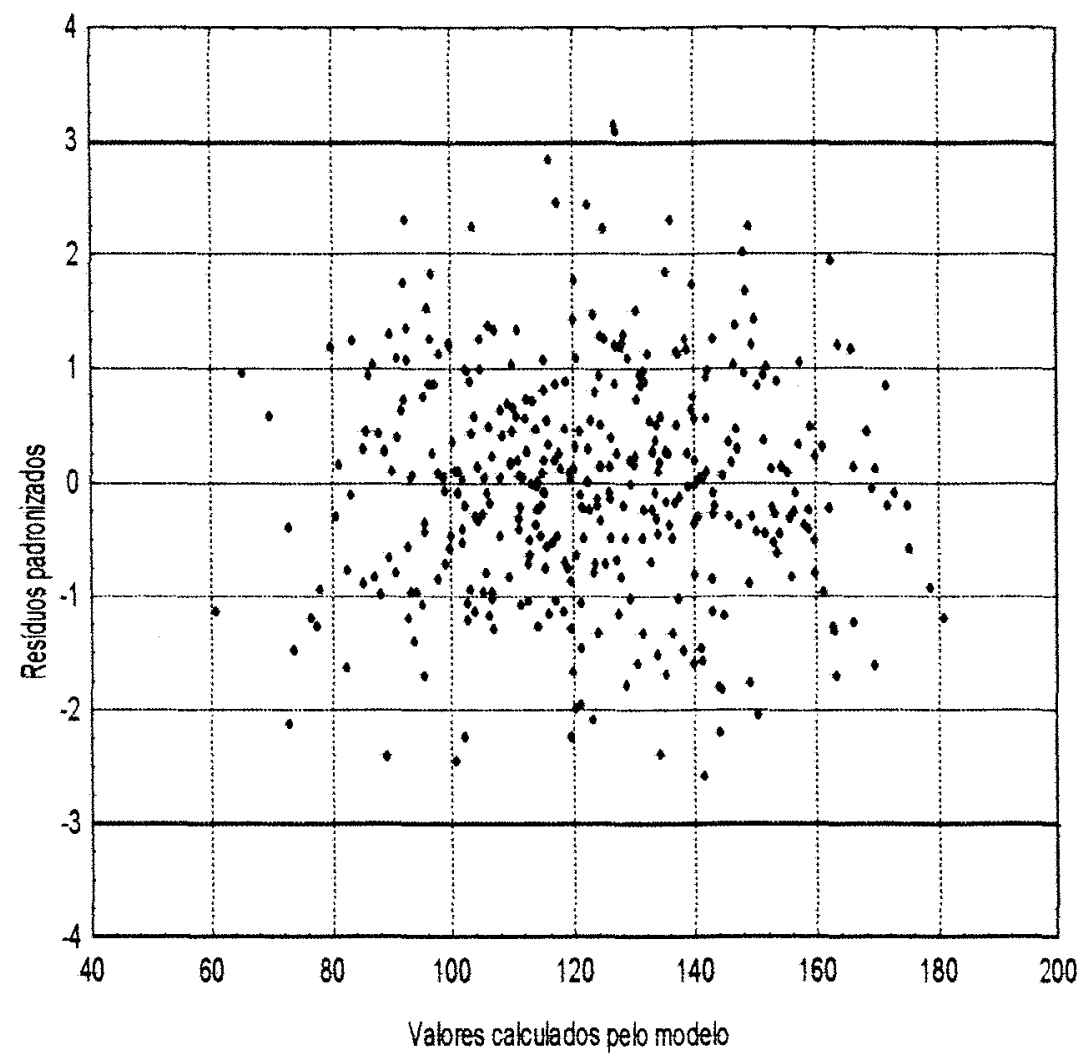

Figura 2: Y(N, K): Dispersão dos resíduos padronizados.

\subsubsection{Estimação e Estudo dos Parâmetros da Superfície Y(N, K)}

Utilizou-se o procedimento REG do SAS para estimar os parâmetros da superfície. A equação obtida foi a seguinte:

$$
Y=108,07+4,57 N+17,72 K-5,61 K^{2}+2,43 N K .
$$

Verifica-se, pois, que há efeito linear positivo de $\mathrm{N}$ e $\mathrm{K}$ e também positivo para a interação $\mathrm{N} \times \mathrm{K}$. A Figura 3 apresenta a superfície obtida. 


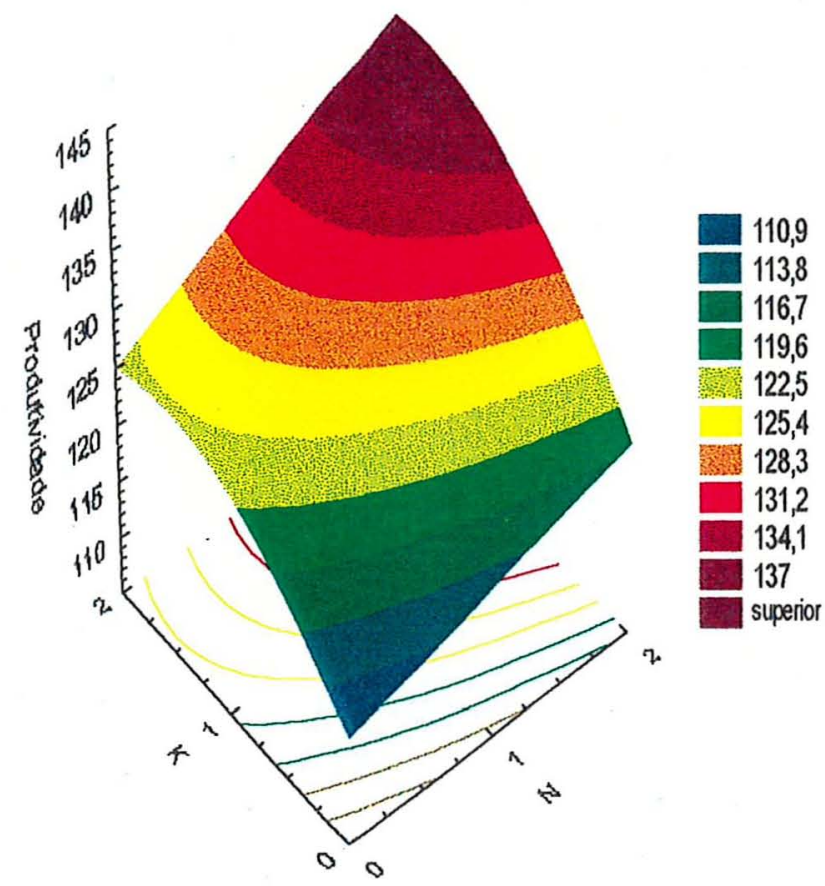

Figura 3: Superfície de resposta para a produtividade de cana-de-açúcar.

\subsubsection{A Função $G(N, K)$ de Produtividade por Real}

Obtido um polinômio de segundo grau Y satisfatório para o estudo, calculou-se a função de custo unitário de produção pelo quociente da função $\mathbf{Y}$ por uma função linẹar dos custos, neste caso:

$$
D=m+t_{1} N+t_{2} K,
$$

onde $\mathrm{m}$ denota o custo fixo da lavoura e $\mathrm{t}_{i}, \mathrm{i}=1,2$, indica o preço dos respectivos nutrientes. Considerando o custo da lavoura de $\mathrm{R} \$ 550$ /ha, a função $\mathrm{D}$ é:

$$
D=550+59,26 N+44,67 K,
$$


e, portanto, a função $\mathrm{G}(\mathrm{N}, \mathrm{K})$ de custo unitário de produção é:

$$
G(N, K)=\frac{Y}{D}=\frac{108,07+4,57 N+17,72 K-5,61 K^{2}+2,43 N K}{550+59,26 N+44,67 K}[t / \text { Real }] .
$$

Para estimar o ponto de máximo da função $G(N, K)$ utilizou-se um processo iterativo, que consistiu no ajuste de um polinômio de segundo grau a uma malha de pontos pertencentes à função a estudar. Neste caso, a malha foi construída com os nove pontos correspondentes a todas as combinações das doses $0 ; 1$ e 2 de ambos nutrientes. A tabela seguinte apresenta esses valores:

Tabela 7: Valores de G para os níveis de N e K.

\begin{tabular}{|ccc|ccc|ccc|}
\hline $\mathrm{N}$ & $\mathrm{K}$ & Valor de $\mathrm{G}$ & $\mathrm{N}$ & $\mathrm{K}$ & Valor de $\mathrm{G}$ & $\mathrm{N}$ & $\mathrm{K}$ & Valor de $\mathrm{G}$ \\
\hline 0 & 0 & 0,1965 & 1 & 0 & 0,1849 & 2 & 0 & 0,1753 \\
0 & 1 & 0,2021 & 1 & 1 & 0,1945 & 2 & 1 & 0,1881 \\
0 & 2 & 0,1894 & 1 & 2 & 0,1868 & 2 & 2 & 0,1846 \\
\hline
\end{tabular}

A equação polinomial de segundo grau $H_{1}(N, K)$ (o índice 1 indica que a equação corresponde à primeira iteração), obtida através do procedimento REG do SAS, é

$$
H_{1}=0,196-0,011 N+0,014 K-0.009 K^{2}+0.004 N K,
$$

representada na Figura 4.

Confirma-se visualmente que o ponto de máximo da função encontrase fora da região experimental e que o máximo absoluto encontrar-se-á na fronteira definida pelo nível zero do nitrogênio. 


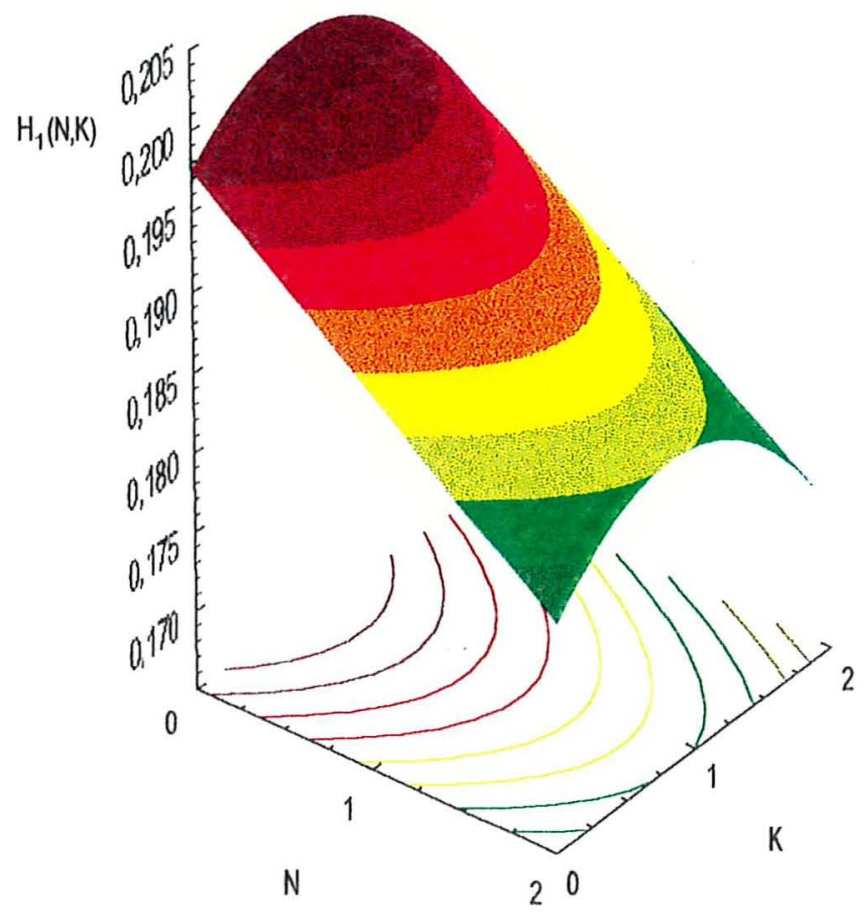

Figura 4: Superfície $\mathrm{H}_{1}(\mathrm{~N}, \mathrm{~K})$ que aproxima $\mathrm{G}(\mathrm{N}, \mathrm{K})$ na região experimental.

Uma advertência sobre o procedimento RSREG

Este procedimento só ajusta superfícies de resposta quadráticas completas, desconsiderando o fato de que nem sempre todos os efeitos quadráticos são significativos, o que se torna uma desvantagem no momento de ajustar um modelo mais simples. Este problema tem consequências na estimação dos parâmetros dos efeitos lineares, na avaliação do ajuste do modelo e nos resultados da análise canônica.

Se, por outro lado, um polinômio quadrático completo ajustou-se bem aos dados, mas o ponto crítico de interesse enconcontra-se fora dos limites da região explorada pelo experimento, a obtenção do ponto de máximo absoluto, na fronteira da região experimental, pelo procedimento RSREG não é simples. Deve ser feita 
através da opção ridge, que procura o máximo da função dentro de uma região que pode ser definida pelo usuário. Mediante aproximações sucessivas consegue-se definir uma região que inclua o máximo absoluto.

\subsubsection{Análise Canônica de G(N, K)}

Dado que o único procedimento que realiza análise canônica é o RSREG, que só ajusta polinômios quadráticos completos, decidiu-se realizar a análise canônica da função $H_{1}(N, K)$ através do estudo das suas primeiras e segundas derivadas parciais. Tem-se, assim:

$$
\begin{aligned}
& \frac{\partial H_{1}}{\partial N}=-0,011+0,004 K \\
& \frac{\partial H_{1}}{\partial K}=0,014-0,018 K+0,004 N
\end{aligned}
$$

Estas funçōes, quando igualadas a zero, fornecem o ponto crítico

$$
P(N=8,875 ; \quad K=2,75) .
$$

A matriz Hessiana é:

$$
\left(\begin{array}{cc}
0 & 0,004 \\
0,004 & -0,018
\end{array}\right),
$$

a qual indica que o ponto achado não corresponde a um ponto de máximo, de mínimo ou de sela. Nesta situação deve-se procurar o máximo absoluto na fronteira da região experimental. Convém salientar que a função $\mathrm{H}$ obtida pelo RSREG possui ponto de sela, que não se corresponde com a análise canônica apresentada.

Pela Figura 4, percebe-se que a região do máximo absoluto encontrase na fronteira da região experimental limitada pelo nível zero do fator nitrogênio. 
Este fato permite obter facilmente o ponto de máximo absoluto, já que a função a maximizar reduz-se a

$$
H_{1}(N=0 ; K)=0,196+0,014 K-0,009 K^{2} .
$$

Esta função possui um máximo em $\mathrm{K}=0,78$, portanto, o ponto de máximo absoluto é o ponto

$$
P(N=0 ; K=0,78) \text {. }
$$

No entanto, o ponto de máximo achado corresponde apenas aproximadamente ao máximo da função $\mathrm{G}(\mathrm{N}, \mathrm{K})$. Portanto, fez-se uma segunda iteração para estimar com maior exatidão esse ponto. Para isso, criou-se uma nova malha de pontos esperados da função na vizinhança do ponto $\mathrm{P}(\mathrm{N}=0 ; \mathrm{K}=0,78)$. Um gráfico esquemático do processo iterativo apresenta-se na Figura 5.

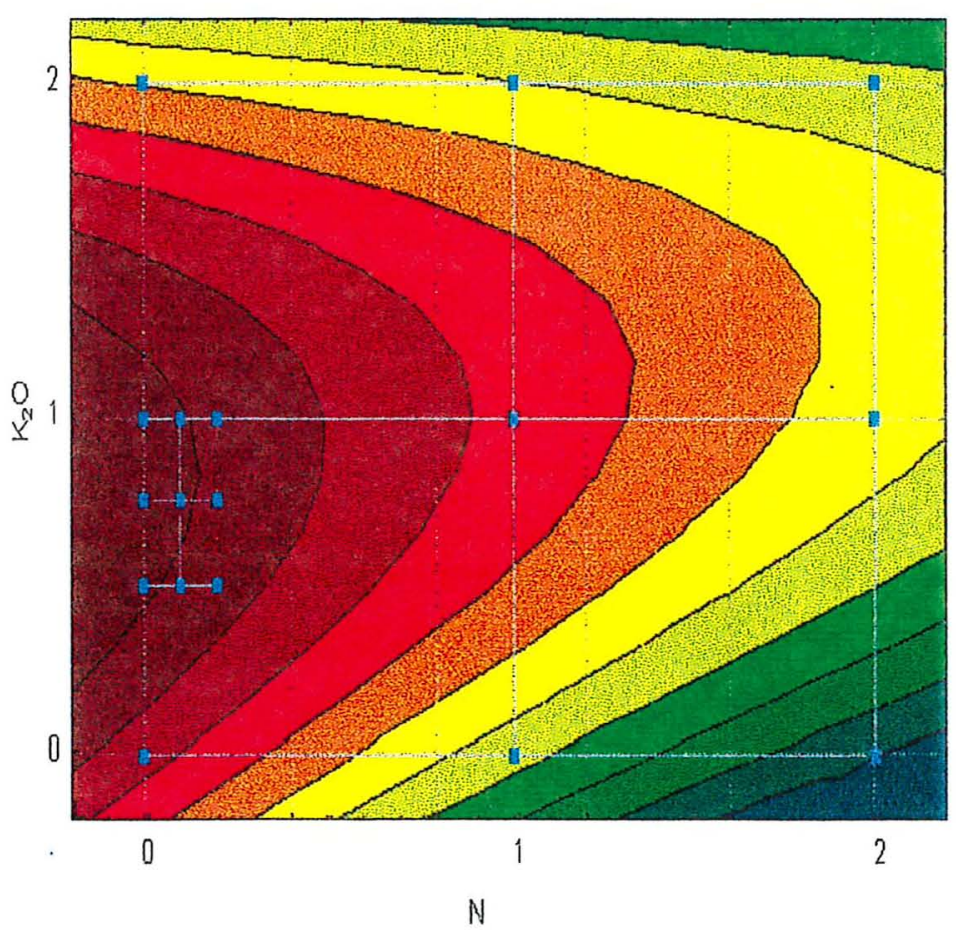

Figura 5: Pontos estimados da função $G(N, K)$. 
Ajustou-se, pois, uma outra superfície $H_{2}(N, K)$ cuja equação,

$$
H_{2}=0,197-0,013 N+0,015 K-0,0096 K^{2}+0,005 N K,
$$

repete quase exatamente a obtida na primeira iteração. Com nova análise canônica, chega-se ao ponto de máximo absoluto fixando em zero o nível de nitrogênio. Assim, $\frac{d H_{2}(K)}{d K}=0,015-0,00192 K=0$ dá $\mathrm{K}=0,78$, e o novo ponto de máximo é:

$$
P(N=0 ; K=0,78) .
$$

Em função dos resultados obtidos, conclui-se que as doses de nutrientes $\mathrm{N}$ e $\mathrm{K}$ recomendáveis para obter o custo mínimo de adubação em cana-de-açúcar, correspondem a $\mathrm{N}=0 ; \mathrm{K}=0,78$. Este último valor equivalente a $58,5 \mathrm{~kg} / \mathrm{ha}$ de $\mathrm{K}_{2} \mathrm{O}$ ou 97,5 $\mathrm{kg} / \mathrm{ha}$ de cloreto de potássio.

Confirma-se que, no estudo com duas variáveis, o ajuste de um polinômio de segundo grau à função $G(N, K)$ é satisfatório e que o procedimento resolveu o problema rapidamente.

\subsection{Estudo com Três Variáveis}

A seguir, tentou-se ajustar um modelo empírico polinomial de segundo grau em função dos níveis de N, P e K. Este tipo de estudo a justa-se melhor aos casos práticos, pois a adubação frequentemente é realizada com base nesses três nutrientes.

\subsubsection{Análise da Variância}

Neste caso, portanto, deve-se verificar a existência de efeitos lineares e quadráticos do fator fósforo além das interações entre ele e os outros nutrientes. A análise da variância apresenta-se na Tabela 8 . 
Detectou-se, além dos efeitos já comprovados no caso de duas variáveis, efeito linear do fator fósforo, mas não foi comprovado o seu efeito quadrático $(\mathrm{F}=0,49)$, que foi eliminado. A interação $\mathrm{N}^{\prime} \times \mathrm{P}^{\prime}$ também não foi significativa $(\mathbf{F}=0,004)$. Mas, existe alguma evidência nos dados de existência das interações $\mathrm{N}^{\prime} \times \mathrm{K}^{\prime}$ e $\mathrm{P}^{\prime} \times \mathrm{K}^{\prime}$, pelo menos ao nível de significancia de $10 \%$. Finalmente, o resíduo, agora com 264 graus de liberdade, permite estimar com maior precisão o efeito da interação tripla, significativa agora ao nível de $0,5 \%$ de probabilidade.

Estes resultados, justificam a utilização da técnica de superfície de resposta para realizar inferências sobre os efeitos dos três nutrientes considerados neste estudo.

Tabela 8: $\mathrm{Y}(\mathrm{N}, \mathrm{P}, \mathrm{K})$ : análise da variância.

\begin{tabular}{|c|c|c|c|c|c|}
\hline $\begin{array}{l}\text { Causa de } \\
\text { Variação }\end{array}$ & G.L. & $\begin{array}{l}\text { Somas de } \\
\text { Quadrados }\end{array}$ & $\begin{array}{c}\text { Quadrados } \\
\text { Médios }\end{array}$ & $\mathbf{F}$ & Probabilidade \\
\hline EXPERIMENTOS (E) & 13 & $157.587,27$ & $12.122,10$ & 16,460 & $0,0001^{* *}$ \\
\hline $\operatorname{BLOCOS}(E)$ & 28 & $10.603,32$ & 378,69 & & \\
\hline $\mathrm{N}^{\prime}$ & 1 & $12.363,40$ & $12.363,40$ & 26,500 & $0,0002^{* *}$ \\
\hline $\mathrm{E} \times \mathrm{N}^{\prime}$ & 13 & $6.064,79$ & 466,52 & & \\
\hline $\mathrm{K}^{\prime}$ & 1 & $20.153,62$ & $20.153,62$ & 55,020 & $0,0001^{* *}$ \\
\hline $\mathrm{E} \times \mathrm{K}^{\prime}$ & 13 & $4.762,07$ & 366,31 & & \\
\hline $\mathrm{K}^{\prime \prime}$ & 1 & $2.642,46$ & $2.642,46$ & 22,760 & $0,0004^{* *}$ \\
\hline $\mathrm{E} \times \mathrm{K}^{\prime \prime}$ & 13 & $1.509,4$ & 116,11 & & \\
\hline $\mathrm{P}^{\prime}$ & 1 & $2.630,64$ & $2.630,64$ & 9,710 & $0,0076^{* *}$ \\
\hline $\mathrm{E} \times \mathrm{P}^{\prime}$ & 13 & $3.522,29$ & 270,94 & & \\
\hline $\mathrm{N}^{\prime} \times \mathrm{K}^{\prime}$ & 1 & 996,21 & 996,21 & 4,440 & $0,0550 \mathrm{~ns}$ \\
\hline $\mathrm{E} \times \mathrm{N}^{\prime} \times \mathrm{K}^{\prime}$ & 13 & $2.913,26$ & 224,10 & & \\
\hline $\mathrm{P}^{\prime} \times \mathrm{K}^{\prime}$ & 1 & 191,78 & 191,78 & 3,510 & $0,0820 \mathrm{~ns}$ \\
\hline $\mathrm{E} \times \mathrm{P}^{\prime} \times \mathrm{K}^{\prime}$ & 13 & 709,35 & 54,56 & & \\
\hline $\mathrm{N} \times \mathrm{P} \times \mathrm{K}$ & 6 & $2.849,34$ & 474,89 & 3,210 & $0,0046^{* *}$ \\
\hline RESÍDUO & 264 & $39.000,88$ & 147,73 & & \\
\hline
\end{tabular}

TOTAL CORRIGIDO $\quad 377 \quad 268.500,10$ 
Foi realizado também o teste de falta de ajuste para o modelo escolhido com os resultados da Tabela 9 .

Tabela 9: $\mathrm{Y}(\mathrm{N}, \mathrm{P}, \mathrm{K})$ : teste de falta de ajuste.

\begin{tabular}{lrrrrr}
\hline \multicolumn{1}{c}{ Causa de } & \multicolumn{2}{c}{ Somas de } & Quadrados & & \\
\multicolumn{1}{c}{ Variação } & G.L. & Quadrados & Médios & F & Probabilidade \\
\hline EXPERIMENTOS (E) & 13 & $157.587,27$ & $12.122,098$ & 16,46 & $0,0001^{* *}$ \\
BLOCOS(E) & 28 & $10.603,32$ & 378,690 & & \\
\hline REGRESSÃO POL. & 6 & $38.978,13$ & $6.663,02$ & 36,47 & $0,0001^{* *}$ \\
DESVIOS DE REG. & 18 & $4.335,32$ & 240,85 & 1,32 & $0,173 \mathrm{~ns}$ \\
ERRO PURO & 312 & $56.996,06$ & 182,68 & & \\
\hline TOTAL & 377 & $268.500,10$ & & &
\end{tabular}

O modelo polinomial escolhido ajustou-se muito bem aos dados, pois a falta de ajuste não foi significativa. $O$ coeficiente de determinação é $\mathbf{R}^{2}=0,90$.

\subsubsection{Estimação e Estudo dos Parâmetros da Superfície Y(N, P, K)}

A equação considerada, levando em conta a significância dos contrastes na análise da variância, é a seguinte:

$$
Y=103,77+4,57 N+4,30 P+18,79 K-5,61 K^{2}+2,43 N K-1,07 P K .
$$

Dado que só existem efeitos lineares do nitrogênio e do fósforo, ambos positivos, isso implica que o rendimento aumenta conforme crescem as doses desses nutrientes, fato que somente é válido nos intervalos das doses consideradas, já que é sabido que a produtividade atinge um máximo e depois começa a cair devido a limitações biológicas.

A Figura 6 apresenta a distribuição dos resíduos padronizados em função dos valores calculados pelo modelo. 


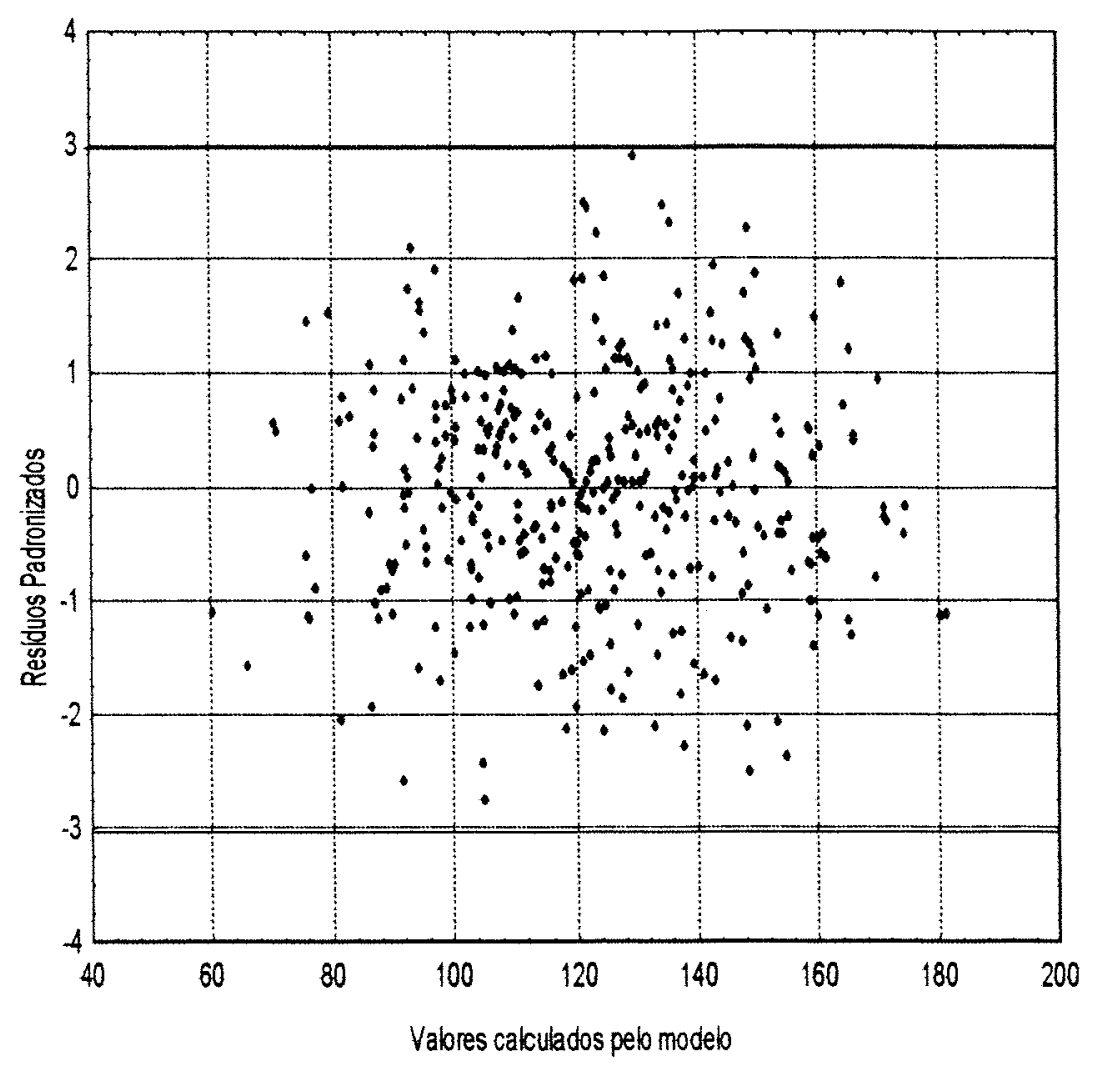

Figura 6: $\mathrm{Y}(\mathrm{N}, \mathrm{P}, \mathrm{K})$ : Dispersão dos resíduos padronizados

\subsubsection{A Função G(N, P, K) de Produtividade por Real}

Neste caso, ela tem a expressão:

$G(N, P, K)=\frac{103,77+4,57 N+4,23 P+18,79 K-5,61 K^{2}+2,43 N K-1,07 P K}{550+59,26 N+85,42 P+44,67 K}$.

Definida a função de interesse, estimaram-se os pontos da região experimental considerada (Tabela 10). 
Tabela 10: Valores de G para os níveis de N, P e K.

\begin{tabular}{|cccc|cccc|cccc|}
\hline $\mathrm{N}$ & $\mathrm{P}$ & $\mathrm{K}$ & Valores de $\mathrm{G}$ & $\mathrm{N}$ & $\mathrm{P}$ & $\mathrm{K}$ & Valores de $\mathrm{G}$ & $\mathrm{N}$ & $\mathrm{P}$ & $\mathrm{K}$ & Valores de $\mathrm{G}$ \\
\hline 0 & 0 & 0 & 0,1887 & 1 & 0 & 0 & 0,1778 & 2 & 0 & 0 & 0,1689 \\
0 & 0 & 1 & 0,1967 & 1 & 0 & 1 & 0,1895 & 2 & 0 & 1 & 0,1836 \\
0 & 0 & 2 & 0,1860 & 1 & 0 & 2 & 0,1837 & 2 & 0 & 2 & 0,1818 \\
0 & 1 & 0 & 0,1701 & 1 & 1 & 0 & 0,1621 & 2 & 1 & 0 & 0,1555 \\
0 & 1 & 1 & 0,1767 & 1 & 1 & 1 & 0,1720 & 2 & 1 & 1 & 0,1680 \\
0 & 1 & 2 & 0,1670 & 1 & 1 & 2 & 0,1664 & 2 & 1 & 2 & 0,1659 \\
0 & 2 & 0 & 0,1559 & 1 & 2 & 0 & 0,1499 & 2 & 2 & 0 & 0,1448 \\
0 & 2 & 1 & 0,1612 & 1 & 2 & 1 & 0,1581 & 2 & 2 & 1 & 0,1554 \\
0 & 2 & 2 & 0,1521 & 1 & 2 & 2 & 0,1526 & 2 & 2 & 2 & 0,1530 \\
\hline
\end{tabular}

\subsubsection{Análise Canônica de G(N, P, K)}

Realizou-se, então, a primeira iteração. Pelo procedimento REG, o polinômio obtido foi:

$$
\begin{aligned}
H_{1}(N . P, K)= & 0,1883-0,0095 N-0,0199 P+0,0149 K+0,0018 P^{2} \\
& -0,0078 K^{2}+0,0017 N P+0,0034 N K-0,0007 P K .
\end{aligned}
$$

Os gráficos de contorno da Figura 7 permitem visualizar o comportamento da função, cujo ponto de máximo, se existir, estará fora da região experimental, e que o ponto de máximo absoluto encontra-se na fronteira limitada pelos níveis $\mathrm{N}=0 \mathrm{e} \mathrm{P}=0$. Este fato obriga a estudar as derivadas da função $H_{1}(N, P, K)$, já que o RSREG não fornece a análise canônica apropriada, como foi explicado no estudo com duas variáveis.

Primeiro, determinou-se o ponto crítico da função, igualando a zero as derivadas parciais: 


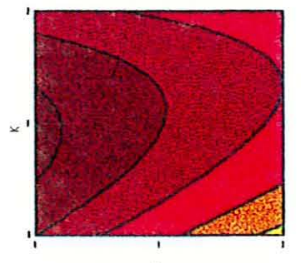

$p=0$

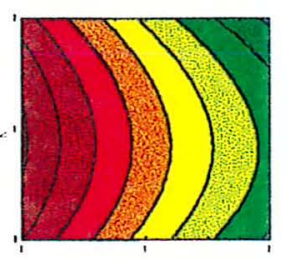

$N=0$

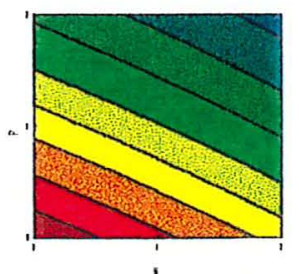

$K=0$

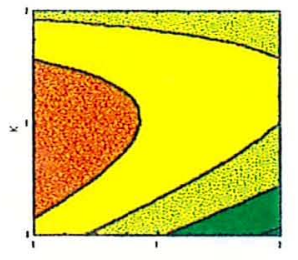

$p=1$

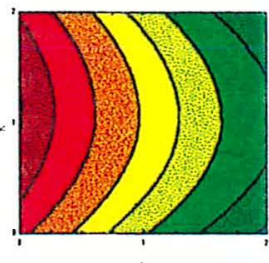

$N=1$

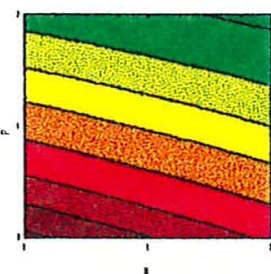

$k=1$

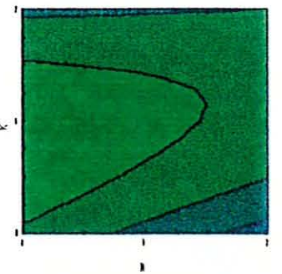

$p=2$

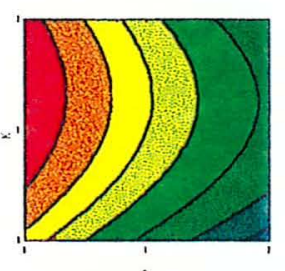

$N=2$

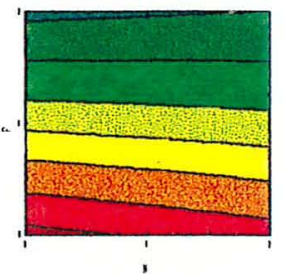

$k=2$

Figura 7: Gráficos de contorno do polinômio $\mathrm{H}_{1}(\mathrm{~N}, \mathrm{P}, \mathrm{K})$

$$
\begin{aligned}
& \frac{\partial H_{1}}{\partial N}=-0,0095+0,0017 P+0,0034 K=0, \\
& \frac{\partial H_{1}}{\partial P}=-0,0199+0,0017 N+0,0036 P-0,0007 K=0, \\
& \frac{\partial H_{1}}{\partial K}=0,0149+0,0034 N-0,0007 P-0,0156 K=0 .
\end{aligned}
$$

$\mathrm{O}$ ponto achado foi $\mathrm{P}(\mathrm{N}=-30,778 ; \mathrm{P}=18,779 ; \mathrm{K}=-6,595)$.

A matriz Hessiana é:

$$
\left(\begin{array}{lrr}
0 & 0,0017 & 0,0034 \\
0,0017 & 0,0036 & -0,0007 \\
0,0034 & -0,0007 & -0,0156
\end{array}\right),
$$


o que demonstra que esta superfície não tem ponto de mínimo, de máximo ou de sela. Nesta situação, deve-se procurar o máximo absoluto na fronteira da região experimental.

À vista da Figura 7, percebe-se que o máximo absoluto estará definido pelos níveis $\mathrm{N}=0$ e $\mathrm{P}=0$. Assim, a função a maximizar reduz-se a:

$$
H_{1}=0,189+0,0141 K-0,0078 K^{2},
$$

que apresenta um ponto de máximo em $\mathrm{K}=0,90$. Portanto, o ponto de máximo absoluto é

$$
P(N=0 ; P=0 ; K=0,90) \text {. }
$$

\section{Segunda Iteração}

Criou-se uma malha de pontos na vizinhança do ponto de máximo obtido. Neste caso foi utilizada a malha resultante da combinação dos valores 0; 0,05 e 0,10 de $\mathrm{N}$ e $\mathrm{P}$, e 0,5; 1 e 1,5 de K. A Figura 8 apresenta esquematicamente a estratégia seguida no processo. O polinômio obtido foi

$$
\begin{aligned}
H_{2}(N, P, K)= & 0,1897-0,0127 N-0,0233 P+0,0159 K+0,0007 N^{2}+0,0031 P^{2} \\
& -0,0090 K^{2}+0,0032 N P+0,0049 N K+0,0004 P K .
\end{aligned}
$$

Com $\mathrm{N}=0$ e $\mathrm{P}=0$, o ponto de máximo absoluto é

$$
P(N=0 ; P=0 ; K=0,88) .
$$




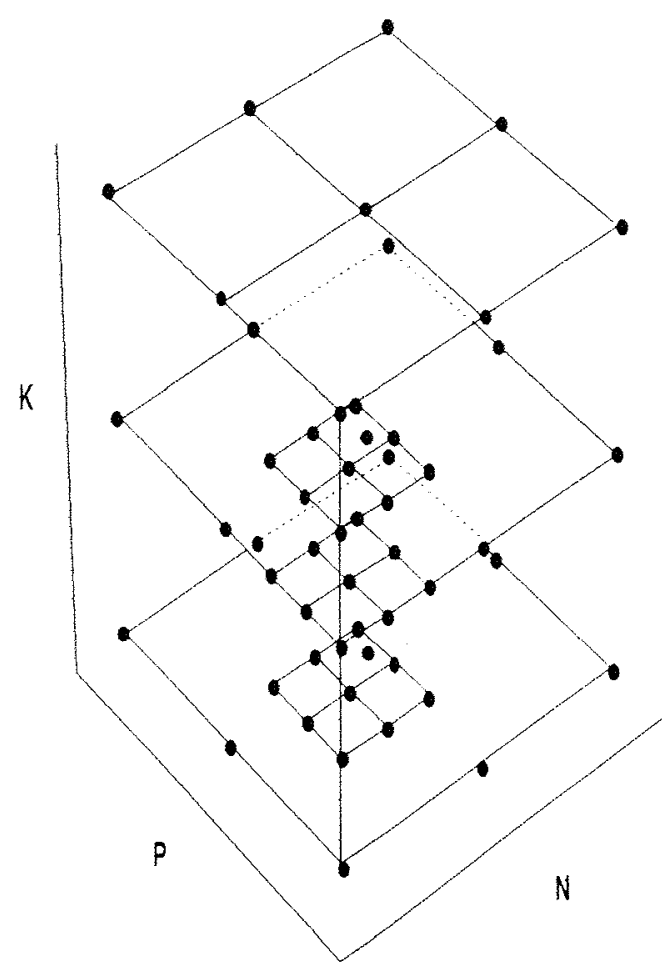

Figura 8: Esquema da estratégia seguida no processo iterativo.

\section{Terceira Iteração}

Neste caso, tem-se

$$
\begin{aligned}
H_{3}(N, P . K)= & 0,1893-0,0125 N-0,0228 P+0,0167 K+0,0008 N^{2}+0,0032 P^{2} \\
& -0,0094 K^{2}+0,0034 N P+0,0047 N K+0,0001 P K,
\end{aligned}
$$

cujo ponto de máximo absoluto é $\mathrm{P}(\mathrm{N}=0 ; \mathrm{P}=0 ; \mathrm{K}=0,89)$.

Em função destes resultados, conclui-se que a dose de adubação que minimiza o custo unitário de produção é dada pelo ponto

$$
P(N=0 ; \quad P=0 ; \quad K=0,89) .
$$


Esta solução equivale a $0 ; 0$ e $66,7 \mathrm{~kg}$ de $\mathrm{N}, \mathrm{P}_{2} \mathrm{O}_{5}$ e $\mathrm{K}_{2} \mathrm{O}$, respectivamente, que corresponde, pela sua vez a $0 ; 0$ e $112,5 \mathrm{~kg}$ de uréia, superfosfato simples e cloreto de potássio, respectivamente.

\subsection{Validação}

$\mathrm{O}$ valor da função $\mathrm{G}$ calculado no ponto $\mathrm{P}(\mathrm{N}=0 ; \mathrm{P}=0 ; \mathrm{K}=0,89)$ é 0,197 t/real. Pela Tabela 10, pode-se conferir que o processo atingiu o máximo absoluto. 


\section{CONCLUSÕES}

- A geração de uma função $G$ de produtividade por real parece ser uma boa estratégia para encarar o problema da minimização do custo do produto agrícola em ensaios de adubação mineral.

- Esta metodologia permite, por um lado, determinar que efeitos são importantes e devem, portanto, estar presentes na função $\mathrm{G}$ e, por outro, estimar seu ponto de máximo (relativo ou absoluto).

- A metodologia desenvolvida parece resolver apropriadamente o problema da maximização da função $\mathrm{G}$ com duas ou três variáveis, quando se usa um polinômio de segundo grau para representar a produtividade.

- Tanto a função $G(N, K)$ como a $G(N, P, K)$ foram bem aproximadas por um polinômio de segundo grau.

- Se houver ponto de máximo e ele estiver dentro da região experimental, em poucos passos atinge-se o ótimo, através da análise canônica realizada pelo procedimento RSREG do SAS.

- Se houver ponto de mínimo ou ponto de sela, ou ainda, ponto de máximo fora da região explorada pelo experimento, a solução do problema, que seria um máximo absoluto, estará na fronteira dessa região.

- Neste último caso, a análise canônica, feita pelo procedimento RSREG do SAS, não é apropriada, devendo-se realizar através do estudo das derivadas 
do polinômio de segundo grau que aproxima a função $\mathbf{G}$.

- A convergência em ambos os casos (ponto de máximo e ponto de máximo absoluto) foi rápida.

- O procedimento RSREG do SAS, desenvolvido para ajustar polinômios de segundo grau completos, não funciona bem quando há efeitos quadráticos nãosignificativos, o que recomendaria o uso de polinômios incompletos. 


\section{REFERÊNCIAS BIBLIOGRÁFICAS}

BOX, G.E.P. The exploration and exploitation of response surfaces: some general considerations and examples. Biometrics, v.10, p.16-70, March 1954.

BOX, G.E.P.; HUNTER, J.S. Multifactor experimental designs for exploring response surfaces. Annals of Mathematical Statistics, v.28, p.195-241, 1957.

BOX, G.E.P.; WILSON, K.B. On the experimental attainment of optimum conditions. Journal of the Royal Statistical Society, v.13, n.1, p.1-45, 1951.

CAMPOS, H. Aspectos da aplicação das superfícies de resposta a ensaios fatoriais $3^{3}$ de adubação. Piracicaba, 1967. 82p. Tese (Livre Docência) - ESALQ - USP.

COCHRAN, W.G.; COX, G.M. Experimental Designs. 2a . Ed. New York: Wiley, 1957. 611p.

D'ACLÍSIO, M. de B. G. Influência dos erros experimentais sobre as recomendações de adubação obtidas pela lei de Mitscherlich. Piracicaba, 1973. 73p. Tese (Doutorado) - Faculdade de Ciências Mêdicas e Biológicas de Botucatu.

D’Aulísio, M. de B. G. A variância dos pontos de máximo ou de mínimo de equações de regressão de segundo grau. Piracicaba, 1976. 61p. Tese (Mestrado) - ESALQ - USP.

GOMES, M. de B. Intervalos de confiança para as coordenadas do ponto crítico da equação quadrática de regressão a duas variáveis independentes. Piracicaba, 1989. 70p. Tese (Livre Docência) - ESALQ - USP. 
HEADY, E.O.; DILLON, J.L. Agricultural production functions. Iowa: Iowa State University Press, 1961. 667p.

MALAVOLTA, E.; PIMENTEL-GOMES,F.; COURY, T. et al. A diagnose foliar na cana-de-açúcar IV. Resultados de 40 ensaios fatoriais NPK $3 \times 3 \times 3$, primeiro corte no Estado de São Paulo. ESALQ-USP, Piracicaba. 1963. 47p.

MYERS, R.H.; MONTGOMERY, D.C. Response surface methodology: Process and product optimization using designed experiments. New York: John Wiley \& Sons, Inc. 1995. 700p.

JORGE, J. de P. N.; CONAGIN, A. Estudos em um grupo especial de delineamentos $\left(\frac{1}{5}\right)\left(5^{3}\right)$. Bragantia v.36, n.4, p.59-88, fev. 1977.

PIMENTEL-GOMES, F. On a formula for the estimation of the optimum dressing of a fertilizer. Biometrics. v.17, p.492-494. Sep. 1961.

PIMENTEL-GOMES, F.; ABREU, C.P. Sobre uma fórmula para o cálculo da dose mais econômica de adubo. Anais da Escola Superior de Agricultura Luiz de Queiroz. n.16, p.191-198. 1959.

PIMENTEL-GOMES, F.; CONAGIN, A. Experimentos de adubação: planejamento e análise estatística. In: $2^{\circ}$ SIMPÓSIO DE ESTATÍSTICA APLICADA À EXPERIMENTAÇÃO AGRONÔMICA E 32० REUNIÃO ANUAL DA REGIÃO BRASILEIRA DA SOCIEDADE INTERNACIONAL DE BIOMETRIA. Londrina, Paraná, Departamento de Matemática Aplicada, UEL, 1987. Anais. Londrina: Região Brasileira da SIB, 1987. p.102.

PIMENTEL-GOMES, F.; GARCIA, C.H. Produção de madeira de custo mínimo. IPEF, v. 48/49, p.153-156, 1995.

REY PASTOR, J.; CALLEJA, P.; TREJO, C.A. Análisis Matemático: Análisis Algebraico. Teoría de ecuaciones. Cálculo infinitesimal de una variable. 8.ed. Argentina: Editorial Kapelusz S.A. 1969. 3v. 
SAS Institute Inc., SAS/STAT ${ }^{T M}$ User's Guide, Release 6.03 Edition. Cary, NC: SAS Institute Inc., 1988, 1028p.

StatSoft Inc, STATISTICA for Windows. Computer program manual. Tulsa, OK: StatSoft, Inc. 1998. 5v.

VIEIRA, S.; ARRUDA, H.V. de; HOFFMAN, R. Estudo comparativo de três funções na análise econométrica de experimentos de adubação. Piracibaba: Convênio ESCO-IMA ESALQ-USP. 1971. 111p.

ZAGATTO, A.G.; PIMENTEL-GOMES, F. O problema técnico - econômico da adubação. Anais da Escola Superior de Agricultura Luiz de Queiroz. n.17, p.149-163. 1960.

ZAGATTO, A.G.; PIMENTEL-GOMES, F. Princípios de Estatística aplicada à Experimentação com Adubos In: MALAVOLTA, E. Manual de química agrícola: Adubos e adubação. So Paulo: Editora Agronômica Ceres Ltda, 1967. Capítulo 23, p. 500-529: Princípios de Estatística aplicada à Experimentação com Adubos. 


\section{APÊNDICE 1}

Dados de produtividade dos experimentos estudados 
Tabela 11: Rendimentos por experimento (kg/ha).

\begin{tabular}{|c|c|c|c|c|c|c|c|c|c|}
\hline \multicolumn{3}{|c|}{ Nutrientes } & \multicolumn{7}{|c|}{ Experimentos } \\
\hline $\mathrm{N}$ & $\mathrm{P}$ & $K$ & 1 & 2 & 3 & 4 & 5 & 6 & 7 \\
\hline \multirow[t]{9}{*}{0} & 0 & 0 & 108,70 & 77,30 & 118,40 & 143,00 & 81,60 & 99,60 & $\overline{140,90}$ \\
\hline & & 1 & 101,60 & 92,10 & 112,50 & 129,50 & 87,10 & 91,20 & 148,70 \\
\hline & & 2 & 129,10 & 89,30 & 125,90 & 139,50 & 92,80 & 98,20 & 153,20 \\
\hline & 1 & 0 & 93,70 & 61,10 & 105,50 & 117,10 & 80,30 & 65,70 & 178,20 \\
\hline & & 1 & 97,80 & 90,80 & 113,00 & 128,40 & 106,00 & 88,90 & 155,70 \\
\hline & & 2 & 107,30 & 95,50 & 126,10 & 120,50 & 124,60 & 107,70 & 155,50 \\
\hline & 2 & 0 & 73,00 & 105,00 & 128,60 & 102,80 & 115,00 & 67,80 & 152,70 \\
\hline & & 1 & 94,30 & 117,70 & 118,60 & 132,00 & 112,50 & 90,10 & 151,60 \\
\hline & & 2 & 90,90 & 81,10 & 125,70 & 137,10 & 132,80 & 95,90 & 159,30 \\
\hline \multirow[t]{9}{*}{1} & 0 & 0 & 109,60 & 73,30 & 108,40 & & 113,20 & 61,10 & 163,60 \\
\hline & & 1 & 118,40 & 112,50 & 122,10 & 168,70 & 113,20 & 88,70 & 146,10 \\
\hline & & 2 & 122,70 & & 136,10 & 161,40 & 106,40 & 104,80 & 182,50 \\
\hline & 1 & 0 & 99,10 & 108,60 & 113,40 & 159,60 & 99,30 & 75,30 & 174,60 \\
\hline & & 1 & 119,60 & 91,10 & 142,10 & 150,90 & 112,00 & 120,90 & 173,90 \\
\hline & & 2 & 141,20 & 122,70 & 132,50 & 153,90 & 108,40 & 105,30 & 168,60 \\
\hline & 2 & 0 & 86,80 & 83,20 & 138,60 & 147,30 & 81,20 & 72,30 & 145,50 \\
\hline & & 1 & 119,80 & 123,60 & 133,40 & 155,20 & 107,10 & 101,60 & 181,20 \\
\hline & & 2 & 150,30 & 113,90 & 144,60 & 149,80 & 109,80 & 104,80 & 167,70 \\
\hline \multirow[t]{9}{*}{2} & 0 & 0 & 99,60 & 91,40 & 131,10 & 134,60 & 115,30 & 76,10 & 153,40 \\
\hline & & 1 & 145,20 & 128,20 & 143,70 & 150,30 & 103,60 & 89,80 & 171,80 \\
\hline & & 2 & 147,30 & 120,30 & 120,90 & 145,90 & 124,10 & 114,30 & 167,80 \\
\hline & 1 & 0 & 105,90 & 93,90 & 124,30 & 105,50 & 100,20 & 61,10 & 145,20 \\
\hline & & 1 & 136,60 & 130,20 & 133,40 & 161,20 & 112,00 & 98,90 & 171,20 \\
\hline & & 2 & 141,40 & 113,70 & 142,50 & 162,80 & 119,30 & 111,80 & 165,50 \\
\hline & 2 & 0 & 149,10 & 96,40 & 124,60 & 135,30 & 108,90 & 110,30 & 154,30 \\
\hline & & 1 & 120,50 & 115,30 & 142,00 & 148,20 & 119,30 & 100,10 & 172,50 \\
\hline & & 2 & 135,20 & 129,80 & 128,40 & 156,20 & 121,40 & 121,40 & 166,60 \\
\hline
\end{tabular}




\begin{tabular}{|c|c|c|c|c|c|c|c|c|c|}
\hline \multicolumn{10}{|c|}{ continuação da página anterior } \\
\hline \multicolumn{3}{|c|}{ Nutrientes } & \multicolumn{7}{|c|}{ Experimentos } \\
\hline $\mathrm{N}$ & $\mathrm{P}$ & $\mathrm{K}$ & 8 & 9 & 10 & 11 & 12 & 13 & 14 \\
\hline \multirow[t]{9}{*}{0} & \multirow[t]{3}{*}{0} & 0 & 102,00 & 80,70 & 112,10 & 152,00 & 142,10 & 90,20 & $\overline{76,80}$ \\
\hline & & 1 & 111,10 & 92,30 & 119,80 & 103,20 & 122,10 & 122,50 & 45,20 \\
\hline & & 2 & 127,70 & 100,30 & 143,20 & 119,30 & 150,00 & 132,10 & 102,30 \\
\hline & \multirow[t]{3}{*}{1} & 0 & 95,20 & 93,20 & 120,00 & 154,10 & 143,20 & 118,70 & 45,90 \\
\hline & & 1 & 154,10 & 111,20 & 142,50 & 154,10 & 165,20 & 115,90 & 102,50 \\
\hline & & 2 & 102,10 & 124,30 & 136,60 & 123,70 & 115,70 & 121,60 & 75,50 \\
\hline & \multirow[t]{3}{*}{2} & 0 & 104,60 & 99,80 & 115,90 & 167,80 & 162,50 & 103,70 & 57,80 \\
\hline & & 1 & 143,40 & 106,10 & 133,70 & 165 & 159,60 & 109,30 & 85,50 \\
\hline & & 2 & 125,50 & 102,10 & 167,00 & 138,90 & 149,30 & 142,40 & 54,50 \\
\hline \multirow[t]{9}{*}{1} & \multirow[t]{3}{*}{0} & 0 & 120,70 & 116,20 & 90,20 & 114,10 & 120,70 & 115,20 & 94,30 \\
\hline & & 1 & 130,50 & 115,90 & 109,60 & 153,00 & 144,60 & 124,80 & 115,70 \\
\hline & & 2 & 126,20 & 116,10 & 130,20 & 145,50 & 145,60 & 123,60 & 81,80 \\
\hline & \multirow[t]{3}{*}{1} & 0 & 103,40 & 99,10 & 120,20 & 143,20 & 138,90 & 97,50 & 73,70 \\
\hline & & 1 & 95,70 & 94,30 & 136,60 & 107,70 & 160,70 & 129,30 & 95,30 \\
\hline & & 2 & 113,40 & 114,10 & 152,30 & 145,50 & 187,70 & 147,80 & 108,60 \\
\hline & \multirow[t]{3}{*}{2} & 0 & 104,30 & 122,50 & 109,60 & 138,20 & 138,40 & 112,30 & 77,80 \\
\hline & & 1 & 143,40 & 118,40 & 114,10 & 148,40 & 165,20 & 166,40 & 116,40 \\
\hline & & 2 & 140,00 & 100,70 & 129,60 & 149,60 & 165,20 & 132,00 & 114,60 \\
\hline \multirow[t]{9}{*}{2} & \multirow[t]{3}{*}{0} & 0 & 113,70 & 100,20 & 96,20 & 125,00 & 118,90 & 108,20 & 77,30 \\
\hline & & 1 & 138,60 & 119,30 & 141,60 & 146,20 & 150,00 & 142,10 & 117,80 \\
\hline & & 2 & 149,80 & 115,90 & 139,50 & 148,40 & 140,90 & 142,00 & 122,00 \\
\hline & \multirow[t]{3}{*}{1} & 0 & 122,00 & 69,10 & 106,80 & 131,10 & 126,10 & 131,20 & 112,80 \\
\hline & & 1 & 128,40 & 119,80 & 124,10 & 179,30 & 171,10 & 130,00 & 93,60 \\
\hline & & 2 & 140,50 & 98,00 & 142,00 & 155,90 & 165,00 & 154,80 & 106,60 \\
\hline & \multirow[t]{3}{*}{2} & 0 & 111,10 & 115,50 & 134,60 & 160,00 & 140,20 & 152,80 & 106,60 \\
\hline & & 1 & 144,10 & 108,90 & 142,80 & 170,20 & 156,20 & 119,10 & 100,30 \\
\hline & & 2 & 129,50 & 114,30 & 164,60 & 153,30 & 169,10 & 137,50 & 126,60 \\
\hline
\end{tabular}

